\title{
CARBON IN RED GIANTS IN GLOBULAR CLUSTERS AND DWARF SPHEROIDAL GALAXIES*
}

\author{
Evan N. Kirby ${ }^{1}$, Michelle Guo ${ }^{2,3}$, Andrew J. Zhang ${ }^{4}$, Michelle Deng ${ }^{5}$, Judith G. Cohen ${ }^{1}$, Puragra Guhathakurta ${ }^{6}$, \\ Matthew D. Shetrone ${ }^{7}$, Young Sun LeE ${ }^{8}$, and LuCa Rizzi ${ }^{9}$ \\ ${ }^{1}$ California Institute of Technology, 1200 E. California Blvd., MC 249-17, Pasadena, CA 91125, USA \\ ${ }^{2}$ Irvington High School, 41800 Blacow Rd., Fremont, CA 94538, USA \\ ${ }^{3}$ Stanford University, 450 Serra Mall, Stanford, CA 94305, USA \\ ${ }^{4}$ The Harker School, 500 Saratoga Ave., San Jose, CA 95129, USA \\ ${ }^{5}$ Harvard University, Massachusetts Hall, Cambridge, MA 02138, USA \\ ${ }^{6}$ UCO/Lick Observatory and Department of Astronomy and Astrophysics, University of California, 1156 High St., Santa Cruz, CA 95064, USA \\ ${ }^{7}$ McDonald Observatory, University of Texas at Austin, HC75 Box 1337-MCD, Fort Davis, TX 79734, USA \\ ${ }^{8}$ Chungnam National University, Department of Astronomy and Space Science, Daejeon 305-764, Korea \\ ${ }^{9}$ Keck Observatory, 65-1120 Mamalahoa Hwy, Kamuela, HI 96743, USA \\ Received 2014 December 5; accepted 2015 January 27; published 2015 March 12
}

\begin{abstract}
We present carbon abundances of red giants in Milky Way (MW) globular clusters and dwarf spheroidal galaxies (dSphs). Our sample includes measurements of carbon abundances for 154 giants in the clusters NGC 2419, M68, and M15 and 398 giants in the dSphs Sculptor, Fornax, Ursa Minor, and Draco. This sample doubles the number of dSph stars with measurements of $[\mathrm{C} / \mathrm{Fe}]$. The $[\mathrm{C} / \mathrm{Fe}]$ ratio in the clusters decreases with increasing luminosity above $\log \left(L / L_{\odot}\right) \simeq 1.6$, which can be explained by deep mixing in evolved giants. The same decrease is observed in dSphs, but the initial $[\mathrm{C} / \mathrm{Fe}]$ of the $\mathrm{dSph}$ giants is not uniform. Stars in dSphs at lower metallicities have larger $[\mathrm{C} / \mathrm{Fe}]$ ratios. We hypothesize that $[\mathrm{C} / \mathrm{Fe}]$ (corrected to the initial carbon abundance) declines with increasing $[\mathrm{Fe} / \mathrm{H}]$ due to the metallicity dependence of the carbon yield of asymptotic giant branch stars and due to the increasing importance of SNe Ia at higher metallicities. We also identified 11 very carbonrich giants (eight previously known) in three dSphs. However, our selection biases preclude a detailed comparison to the carbon-enhanced fraction of the MW stellar halo. Nonetheless, the stars with $[\mathrm{C} / \mathrm{Fe}]<+1$ in dSphs follow a different $[\mathrm{C} / \mathrm{Fe}]$ track with $[\mathrm{Fe} / \mathrm{H}]$ than the halo stars. Specifically, $[\mathrm{C} / \mathrm{Fe}]$ in $\mathrm{dSphs}$ begins to decline at lower $[\mathrm{Fe} / \mathrm{H}]$ than in the halo. The difference in the metallicity of the $[\mathrm{C} / \mathrm{Fe}]$ "knee" adds to the evidence from $[\alpha / \mathrm{Fe}]$ distributions that the progenitors of the halo had a shorter timescale for chemical enrichment than the surviving dSphs.
\end{abstract}

Key words: galaxies: abundances - galaxies: dwarf - Local Group - stars: abundances - stars: evolution

Supporting material: machine-readable tables

\section{INTRODUCTION}

Variations of elemental abundances within a stellar system are evidence for physical processes both internal and external to individual stars. Chemical evolution on a galactic scale leads to a dispersion in metallicity. As progressive generations of supernovae $(\mathrm{SNe})$ enrich the interstellar medium of a galaxy, stars that form later have higher metallicity than their older predecessors (Tinsley 1980). On the other hand, variations in certain light elements can arise from internal stellar evolution. Gravitational settling and radiative levitation (Behr et al. 1999) deplete and enhance, respectively, the abundance of an element in the photosphere of a star relative to the rest of the star. Nuclear burning at the base of a convective zone that reaches the photosphere can also decrease or increase the observable abundances of light elements, like Li (Lind et al. 2011), C, N, $\mathrm{O}$, and even $s$-process elements, like $\mathrm{Ba}$ and $\mathrm{Rb}$ (Liang et al. 2000; Karakas et al. 2012). This process is called astration when nuclear burning leads to the destruction of an element.

Carbon exhibits abundance variations within a stellar population due to astration and galactic chemical evolution.

\footnotetext{
* The data presented herein were obtained at the W. M. Keck Observatory, which is operated as a scientific partnership among the California Institute of Technology, the University of California and the National Aeronautics and Space Administration. The Observatory was made possible by the generous financial support of the W. M. Keck Foundation.
}

As a star ascends the red giant branch (RGB), it encounters episodes of mixing that bring material processed through the CNO cycle to the photosphere. Within 2-3 mag of the tip of the $\mathrm{RGB}, \mathrm{C}$ and $\mathrm{O}$ are depleted and $\mathrm{N}$ is enhanced relative to stars on the main sequence or the lower RGB. Proposed mechanisms for mixing deep CNO-processed material into the upper atmosphere include Rayleigh-Taylor instability (Eggleton et al. 2006) or, more likely, thermohaline mixing (Charbonnel \& Zahn 2007).

Globular clusters (GCs) are excellent sites to study the evolution of carbon. It is relatively easy to test whether variations in carbon abundances within a cluster are primordial or modified by stellar evolutionary processes during the lifetime of the cluster because GCs are essentially single-age, mono-metallic populations at a uniform distance. It is not necessary to control for age or metallicity. Carbon should not be astrated in metal-poor stars on the main sequence and subgiant branch, which do not have convective envelopes deep enough to dredge up CNO-processed material. However, GCs do show primordial abundance variations. Cohen (1999) and Briley et al. (2002) found variations in $[\mathrm{C} / \mathrm{Fe}]$ on the main sequences of M71 and M13, respectively. Because the photospheres of main sequence stars are not affected by convection that can reach CNO-processed material, these carbon variations must be primordial. Regardless, astration on the upper RGB causes the most severe variations (e.g., 
Suntzeff 1981; Smith et al. 1996; Smith \& Briley 2005, 2006). Martell et al. (2008c) showed that the rate of depletion depends on metallicity, as predicted by thermohaline mixing (Charbonnel \& Zahn 2007).

Stars can also become very carbon-rich. "Carbon stars" (see the review by Wallerstein \& Knapp 1998) display extremely strong $\mathrm{C}_{2}, \mathrm{CH}$, and/or $\mathrm{CN}$ bands, and they can be significantly redder than the RGB in optical colors (e.g., Bond \& Neff 1969; Mould \& Aaronson 1979). Carbon can form in helium-burning asymptotic giant branch (AGB) stars through the triple- $\alpha$ process. It can be brought to the surface of an AGB star in the third dredge-up (Iben 1975). This process is most efficient in stars around 2-2.5 $M_{\odot}$ (Mouhcine \& Lançon 2003), which explains why most ancient GCs-where the highest-mass stars are less than $1 M_{\odot}$ - lack C stars (Cohen et al. 1978). C stars also require at least $0.3 \mathrm{Gyr}$ to form (Mouhcine 2002), which makes them good indicators of intermediate-age populations.

Dwarf and RGB stars can acquire carbon through binary mass transfer with a C star (see Lucatello et al. 2005; Cohen et al. 2006; Starkenburg et al. 2014). C-rich RGB stars are rarely seen to have a photometric companion because the AGB donor has almost always evolved into a faint white dwarf long ago. Instead, binarity of C-rich RGB stars is observed spectroscopically through variability in radial velocity (McClure \& Woodsworth 1990). The recipient of carbon through mass transfer can exist in any evolutionary stage, such as the main sequence (Dahn et al. 1977).

Aaronson \& Mould (1980) and Aaronson et al. (1982) used the existence of carbon stars in dwarf spheroidal galaxies (dSphs) to argue for the presence of intermediate-age populations. These stars were selected for spectroscopy from their infrared colors, and their spectra showed very strong $\mathrm{C}_{2}$ bands. However, carbon enhancements need not be so large that they affect broadband colors. For example, Norris et al. (2010) discovered a $\mathrm{C}$-rich $([\mathrm{C} / \mathrm{Fe}]=2.3)$ red giant in the ultra-faint galaxy Segue 1. This star (Segue 1-7) could only have been found spectroscopically because its broadband colors are consistent with carbon-normal RGB stars.

The statistics of carbon abundances in dwarf galaxies are less complete than for GCs or the Milky Way (MW). Although Aaronson \& Mould (1980) and their contemporaries discovered carbon stars in dwarf galaxies, they did not quantify the carbon enhancement. Kinman et al. (1981) published in conference proceedings the first carbon abundances from low-resolution spectroscopy in a dSph, specifically Draco. Smith (1984) followed up with $\mathrm{CH}$ and $\mathrm{CN}$ indices in Draco, and Bell (1985) published more low-resolution $[\mathrm{C} / \mathrm{H}]$ abundances in Draco and Ursa Minor. Shetrone et al. (1998b) later measured low-resolution $[\mathrm{C} / \mathrm{Fe}]$ abundances for two stars in Sculptor, finding one to be mildly carbon-rich. Fulbright et al. (2004) measured the first carbon abundance from highresolution spectroscopy in a dSph, specifically Draco. Abia et al. (2008) measured the ${ }^{12} \mathrm{C} /{ }^{13} \mathrm{C}$ isotopic ratio and the $\mathrm{C} / \mathrm{O}$ ratio-but not $[\mathrm{C} / \mathrm{H}]$-in two carbon stars in Carina. Cohen \& Huang $(2009,2010)$ measured carbon abundances in 18 stars in Draco and Ursa Minor from the $\mathrm{G}$ band of $\mathrm{CH}$, and Skúladóttir et al. (2015) measured C abundances for about 80 Sculptor giants from a near-infrared CN band. Recently, ultrafaint galaxies have received a lot of attention, including carbon abundance measurements in Segue 1 (Norris et al. 2010; Frebel et al. 2014), Ursa Major II and Coma Berenices (Frebel et al. 2010b), Boötes I (Norris et al. 2010; Lai et al. 2011), and
Boötes II (Koch \& Rich 2014). Interestingly, all of these measurements are consistent with GC abundances on the upper RGB except one star in Sculptor (Skúladóttir et al. 2015) and four stars in Segue 1, including its three most metal-poor stars (Norris et al. 2010). The tendency of carbon-rich stars to appear among the metal-poor population led Shetrone et al. (2013) to measure carbon abundances for 35 stars in the metalpoor dSph Draco \& Frebel et al. (2010a) and Starkenburg et al. (2013) to explicitly target the most metal-poor stars in Sculptor. Still, Segue 1 and Sculptor contain the only examples of dwarf galaxy stars with quantified high carbon abundances. (As discussed above, $\mathrm{C}$ stars still exist in dSphs.)

The MW halo contains a large number of C-rich stars, and their frequency increases with decreasing metallicity. However, the exact fraction of carbon-enhanced metal-poor (CEMP) stars as a function of metallicity is contentious (Cohen et al. 2005; Marsteller et al. 2005; Frebel et al. 2006; Lucatello et al. 2006; Carollo et al. 2012; Lee et al. 2013; Yong et al. 2013; Placco et al. 2014), partly because the search for low-metallicity stars is fraught with selection biases (e.g., Schörck et al. 2009). Very roughly, the fraction of stars with $[\mathrm{Fe} / \mathrm{H}]<-2.5$ that are carbon-enhanced is about $20 \%$.

The MW halo is expected to be at least partly composed of the disrupted dSphs from past accretion events (Searle \& Zinn 1978). One apparent challenge to this idea was the discrepancy between the $[\alpha / \mathrm{Fe}]$ ratio of halo and dSph stars at $[\mathrm{Fe} / \mathrm{H}] \gtrsim-2$ (Shetrone et al. 1998a, 2001, 2003; Venn et al. 2004). However, Robertson et al. (2005) and Font et al. (2006) showed that the discrepancy is in fact an expected outcome of hierarchical assembly. The inner halo, where most of the observed halo stars lie, is composed of more massive satellites that do not resemble the present-day, surviving dSphs. At low enough metallicities, most elemental abundance ratios between dwarf galaxies and the MW halo agree (e.g., Frebel et al. 2010b), though the neutron-capture elements are deficient in ultra-faint dwarf galaxies compared to the halo, even at very low metallicities (e.g., Koch et al. 2013).

The fact that so few carbon-rich stars have been found in dSphs, even at low metallicities, might be troublesome for the theory of the hierarchical assembly of the MW halo. The fraction of CEMP stars in Draco, Ursa Minor, and Sculptor is around $10 \%$ or less (Cohen \& Huang 2009, 2010; Shetrone et al. 2013; Starkenburg et al. 2013; Skúladóttir et al. 2015). However, this number does not include C-rich stars for which carbon abundances were not quantified.

In this work, we add to the body of carbon abundance measurements in dSphs. We measured carbon abundances of 398 red giants (eight previously known) in the dSphs Sculptor, Fornax, Ursa Minor, and Draco. We also identified 11 giants that are too carbon-rich to quantify their abundances. The stars observed here are a subset of the stars observed by Kirby et al. (2009, 2010), who measured $\mathrm{Mg}, \mathrm{Si}, \mathrm{Ca}$, Ti, and $\mathrm{Fe}$ abundances in about 3000 red giants in eight dSphs.

\section{SPECTROSCOPIC OBSERVATIONS}

We obtained Keck/DEIMOS (Faber et al. 2003) spectra of the carbon G band in red giants in MW GCs and dSphs. The GCs are NGC 2419, NGC 4590 (M68), and NGC 7078 (M15). The dSphs are Sculptor, Fornax, Ursa Minor, and Draco. We used the same dSph slitmasks as Kirby et al. (2009, 2010), who obtained redder DEIMOS spectra for measuring $\mathrm{Mg}, \mathrm{Si}, \mathrm{Ca}, \mathrm{Ti}$, and $\mathrm{Fe}$ abundances. We re-observed three of five slitmasks 
Table 1

DEIMOS Observations

\begin{tabular}{|c|c|c|c|c|c|c|c|}
\hline System & Slitmask & \# Targets & Date & Airmass & $\begin{array}{c}\text { Seeing } \\
\left({ }^{\prime \prime}\right)\end{array}$ & $\begin{array}{l}\text { Individual Exposures } \\
\text { (s) }\end{array}$ & $\begin{array}{c}\text { Total Exposure Time } \\
\text { (s) }\end{array}$ \\
\hline NGC 2419 & $\mathrm{n} 2419 \mathrm{~b}$ & 112 & 2012 Mar 19 & 1.1 & 0.8 & $3 \times 900$ & 2700 \\
\hline M68 & $\mathrm{n} 4590 \mathrm{a}$ & 96 & 2014 Feb 2 & 1.6 & 0.8 & $1200+937$ & 2137 \\
\hline$\cdots$ & $7078 \mathrm{e}$ & 167 & $2011 \mathrm{Jul} 30$ & 1.0 & 0.9 & $3 \times 900$ & 2700 \\
\hline Sculptor & bscl1 & 86 & 2011 Jul 31 & 1.7 & 0.8 & $4 \times 1200+900$ & 5700 \\
\hline$\ldots$ & bscl2 & 106 & 2011 Aug 6 & 1.8 & 0.7 & $2 \times 1200+2 \times 840$ & 4080 \\
\hline$\cdots$ & $\ldots$ & $\ldots$ & 2011 Aug 6 & 1.8 & 0.8 & 1200 & $\ldots$ \\
\hline$\ldots$ & $\ldots$ & $\ldots$ & 2011 Aug 7 & 1.9 & 0.8 & $3 \times 1200$ & $\ldots$ \\
\hline Ursa Minor & bumi1 & 125 & 2011 Jul 29 & 1.5 & 0.7 & $4 \times 1200+600$ & 5400 \\
\hline$\cdots$ & bumi2 & 134 & $2011 \mathrm{Jul} 31$ & 1.7 & 0.8 & $4 \times 1200$ & 4800 \\
\hline .. & bumi3 & 137 & 2011 Aug 4 & 1.8 & 0.6 & $4 \times 1200$ & 4800 \\
\hline Draco & bdra1 & 135 & $2011 \mathrm{Jul} 30$ & 1.4 & 1.2 & $5 \times 1200$ & 6000 \\
\hline
\end{tabular}

(198 of 376 member stars) in Sculptor, one of five slitmasks (125 of 675 members) in Fornax, three of four slitmasks (87 of 212 members) in Ursa Minor, and three of eight slitmasks (181 of 298 members) in Draco.

\subsection{Target Selection}

We selected stars for the DEIMOS slitmasks from a variety of photometric catalogs, which provided astrometry, magnitudes, and colors of the red giants that we observed. We used Stetson's (2000) catalog for all three GCs. We supplemented Stetson's list of M68 stars with photometry from Alcaino et al. (1990) and Walker (1994). We used Johnson $V$ and Cousins $I$ magnitudes for all GC stars. We corrected the magnitudes for reddening and extinction based on $E(B-V)$ values of 0.08 , 0.05 , and 0.10 for NGC 2419, M68, and M15, respectively (Harris 1996, updated 2010 ${ }^{10}$ ).

Kirby et al. (2009) described the target selection for Sculptor, and Kirby et al. (2010) detailed the target selection for Fornax, Ursa Minor, and Draco. For Sculptor, we used Westfall et al.'s (2006) photometric catalog in Washington $M$ and $T_{2}$ magnitudes. We used sources from Stetson et al.'s (1989) catalog in Cousins $B$ and Johnson $V$ magnitudes for Fornax. For Ursa Minor, we used Bellazzini et al.'s (2002) VI catalog, and for Draco, we used Ségall et al.'s (2007) gi catalog in the CFHT MegaCam system.

We placed stars with colors and magnitudes appropriate for red giants on the slitmasks. For the GCs, we drew a polygon around the RGB in the color-magnitude diagram (CMD). Stars within the polygon were included in the slitmask. Brighter stars were preferred over fainter stars in the cases where slitmask design constraints forced a choice among multiple red giants. For the dSphs, we selected stars from the CMD with the guidance of theoretical isochrones (see Kirby et al. 2010 for additional details). The Sculptor catalog additionally has DDO21 magnitudes, which we used to discriminate between red giants and foreground dwarf stars (see Kirby et al. 2009).

\footnotetext{
${ }^{10}$ http://physics.mcmaster.ca/ harris/mwgc.dat
}

\subsection{Observations}

Table 1 lists the observation dates and exposure times of each slitmask. We used the $900 Z \mathrm{Z}$ grating with a ruling of 900 lines $\mathrm{mm}^{-1}$ and a blaze wavelength of $5500 \AA$. The grating was tilted such that the blaze wavelength fell on the center of the CCD mosaic. Slits were $0.7^{\prime \prime}$ wide. This configuration gives an approximate wavelength range of $4000-7400 \AA$ at a resolution of $2.1 \AA$ FWHM $(R \sim 2100$ at $4300 \AA)$. The exact wavelength range of each spectrum depends on the location of the slit on the slitmask. The starting and ending wavelengths of the spectra vary by $\sim 500 \AA$ across the slitmask.

We obtained internal flat field and arc lamp exposures. We exposed a quartz lamp three times for $12 \mathrm{~s}$ and six times for $45 \mathrm{~s}$, which is appropriate for the red and blue halves of the CCD mosaic, respectively. We also obtained two sets of arc lamp exposures. First, we obtained simultaneous spectra of $\mathrm{Ne}$, $\mathrm{Ar}, \mathrm{Kr}$, and $\mathrm{Xe}$ lamps for $1 \mathrm{~s}$. This exposure is suitable to calibrate the wavelength solution for the red halves of the spectra. Then, we obtained a single exposure for several lamps. We controlled the amount of flux from each lamp by turning off lamps in the middle of the exposure. The lamps were $\mathrm{Hg}$ (1s), Cd (5s), Kr (6s), Ar (12s), and Zn (12s). This exposure is appropriate for the blue halves of the spectra.

All slitmasks were also observed with the $1200 \mathrm{G}$ grating, which has a groove spacing of $1200 \mathrm{~mm}^{-1}$ and a blaze wavelength of $7760 \AA$. The grating was centered at $7800 \AA$. Table 2 of Kirby et al. (2010) lists all of these observations except for the $\mathrm{n} 2419 \mathrm{~b}$ and $\mathrm{n} 4590 \mathrm{a}$ slitmasks. We observed $\mathrm{n} 2419 \mathrm{~b}$ with the $1200 \mathrm{G}$ grating and this $7800 \AA$ setting on 2012 March 19 for $1800 \mathrm{~s}$, and we observed n4590a on 2011 June 2 for $2400 \mathrm{~s}$.

\subsection{Reductions}

We reduced the raw data with the spec2d pipeline ${ }^{11}$ (Cooper et al. 2012) developed by the DEEP2 survey team (Newman et al. 2013). This software is optimized for extended galaxies observed with the $1200 \mathrm{G}$ grating. We made moderate revisions to the pipeline to make it more suitable for bluer DEIMOS spectra. First, we modified the software to accept separate flat

\footnotetext{
11 http://2.keck.hawaii.edu/inst/deimos/pipeline.html
} 


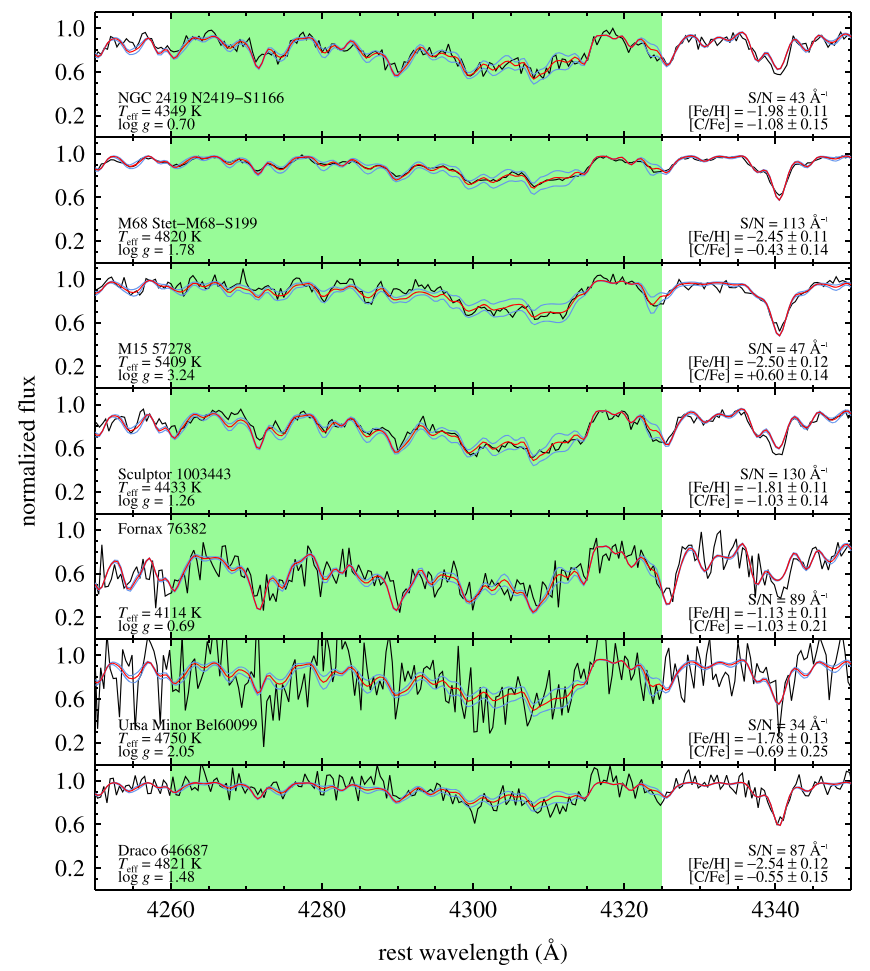

Figure 1. Portions of DEIMOS spectra around the $\mathrm{G}$ band for one star in each of the GCs and dSphs observed. The black line is the observed spectrum, and the red line is the best-fitting synthetic spectrum. The fit was performed in the green shaded region. The pale blue lines show synthetic spectra with $[\mathrm{C} / \mathrm{Fe}] \pm 0.2$ different from the best-fitting value.

lamp exposures for the red and blue CCDs. Second, we revised the arc lamp line lists for $\mathrm{Hg}, \mathrm{Cd}, \mathrm{Kr}, \mathrm{Ar}$, and $\mathrm{Zn}$ between 4158 and $5496 \AA$. We eliminated lines that we could not see in individual DEIMOS arc lamp exposures. Third, we changed the tolerances for automatic identification of arc lines so that the pipeline would include some of the weaker blue lines. Fourth, we changed the way that the two-dimensional spectrum is rectified. The curvature of the focal plane and the slit position angle can introduce a tilt in the spatial direction such that a single wavelength corresponds to a diagonal line rather than a single row of pixels. Previously, this tilt was removed by shifting CCD columns by integer values. We changed the code to allow non-integer shifts, which slightly increased the effective spectral resolution and signal-to-noise ratio $(\mathrm{S} / \mathrm{N})$. Fifth, we implemented a correction for atmospheric dispersion along the slit, which is important for our bluer spectra. Because light at $4000 \AA$ can be displaced along the slit by a few pixels from light at $7400 \AA$, we curved the extraction window to match the shape of the atmospheric dispersion. Finally, we adopted the optimizations for point sources developed by Simon \& Geha (2007).

The results of the reductions were wavelength-calibrated, sky-subtracted, one-dimensional spectra. Cosmic rays were removed before the one-dimensional spectra were extracted. The spec $2 \mathrm{~d}$ code also calculated and saved variance (error) spectra, which we used to estimate random measurement uncertainty on $[\mathrm{C} / \mathrm{Fe}]$ (Section 3.4.1).

Figure 1 shows one example spectrum from each stellar system in Table 1 . The figure shows spectra at a variety of $\mathrm{S} / \mathrm{N}$. Each panel lists the $\mathrm{S} / \mathrm{N}$, which is calculated as the median

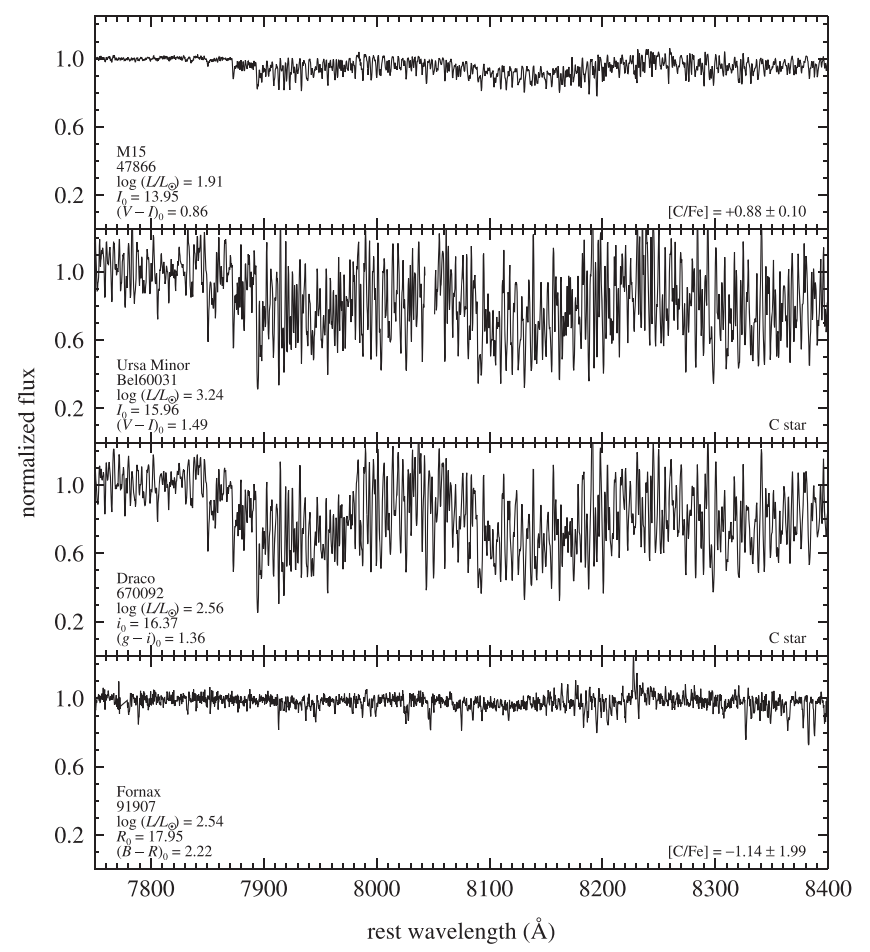

Figure 2. The top panel shows a moderately carbon-rich AGB star in M15. The $\mathrm{CN}$ bandhead is at $\sim 7850 \AA$, and $\mathrm{CN}$ absorption is visible redward of the bandhead. The next two panels show $\mathrm{CN}$ absorption in two of the 11 carbon stars in the DEIMOS sample. The bottom panel shows a carbon-normal RGB star for comparison.

absolute deviation of the observed spectrum (black) from the best-fitting synthetic spectrum (red).

\subsection{Carbon Stars}

We found 11 examples of carbon stars in our sample. The red spectra of these stars are dominated by very strong $\mathrm{CN}$ absorption, which precluded measurement of atmospheric parameters like $[\mathrm{Fe} / \mathrm{H}]$. The $\mathrm{G}$ bands are also strong enough that we would not have found our measurements of carbon abundances reliable due to saturation. Figure 2 shows examples of the near-infrared spectra of some of these stars. We discuss carbon stars further in Sections 5.1 and 6.

\section{SPECTROSCOPIC MEASUREMENTS}

Before we could measure carbon abundances from the spectra, we needed to measure the stars' radial velocities $\left(v_{r}\right)$ and atmospheric parameters.

\subsection{Radial Velocity Measurements}

We previously measured $v_{r}$ for all of the spectra from our red DEIMOS observations (Kirby et al. 2009, 2010). However, the wavelength calibration for the blue DEIMOS spectra is not as precise as the red spectra because of the relative sparsity of blue arc lamp lines. Rather than apply the known $v_{r}$ from the red spectra to the blue spectra, we measured $v_{r}$ directly from the $\mathrm{G}$ band in the blue spectra. Although $v_{r \text {,blue }}$ is not as precise as $v_{r \text {,red }}$ (for example, for measuring galactic velocity dispersions), $v_{r \text {,blue }}$ is more suited to shifting the $\mathrm{G}$ band into the rest frame. 
We measured $v_{r \text {,blue }}$ by cross-correlating the observed spectrum with a synthetic spectrum. The synthetic spectrum had the atmospheric parameters previously measured from the red spectra (see Section 3.2) and $[\mathrm{C} / \mathrm{Fe}]=0.0$. The spectra were synthesized according to the procedure described in Section 3.4. We performed the cross-correlation in the range $4200-4400 \AA$, and we set $v_{r}$ to be the velocity corresponding to the cross-correlation peak. For the remaining analysis, we shifted the spectrum into the rest frame using this measurement of $v_{r}$.

We also evaluated the membership of each star by its radial velocity. (Kirby et al. 2010, their section 2.6) gave the details of the membership criteria. In summary, we eliminated stars more than three standard deviations away from the mean radial velocity.

\subsection{Atmospheric Parameters}

The measurement of carbon abundances (Section 3.4) requires knowledge of effective temperatures $\left(T_{\text {eff }}\right)$, surface gravities $(\log g)$, metallicities $([\mathrm{Fe} / \mathrm{H}])$, and alpha enhancements $([\alpha / \mathrm{Fe}])$. Kirby et al. $(2009,2010)$ measured these parameters for most of the spectra presented here. They obtained preliminary estimates of $T_{\text {eff }}$ and $\log g$ by comparing the stars' colors and magnitudes to theoretical isochrones. They refined $T_{\text {eff }}$ and measured $[\mathrm{Fe} / \mathrm{H}]$ and $[\alpha / \mathrm{Fe}]$ by minimizing $\chi^{2}$ between the observed red DEIMOS spectra and a large grid of synthetic spectra (Kirby 2011).

We adopted without modification the previously determined atmospheric parameters. However, the DEIMOS slitmasks $\mathrm{n} 2419 \mathrm{~b}$ and $\mathrm{n} 4590 \mathrm{a}$ were not included in the previous catalogs of atmospheric parameters. We measured these parameters in the same manner as Kirby et al. (2010).

\subsection{Grid of Synthetic Spectra}

We measured $[\mathrm{C} / \mathrm{Fe}]$ by comparing observed spectra of the $\mathrm{G}$ band to a grid of synthetic spectra spanning 4100-4500 $\AA$. We computed this grid in a similar fashion to the way in which Kirby et al. (2008) computed their synthetic spectral grid spanning 6300-9100 $\AA$ in order to measure $[\mathrm{Fe} / \mathrm{H}]$ and $[\alpha / \mathrm{Fe}]$ from red DEIMOS spectra. First, we compiled a line list from publicly available catalogs of atomic and molecular data. We refined the line list by comparing it to the spectra of the Sun and Arcturus. Then, we computed MOOG synthetic spectra using ATLAS9 model atmospheres.

\subsubsection{Line List}

The $\mathrm{G}$ band has a high density of $\mathrm{CH}$ molecular absorption lines. At the low resolution of our DEIMOS spectra, individual $\mathrm{CH}$ lines are indistinguishable from individual atomic and other molecular lines. Therefore, we needed to model the absorption due to many species, not just $\mathrm{CH}$. We compiled a list of atomic and molecular transitions in the range 4100-4500 A.

We started by including all the neutral and singly ionized atomic transitions listed in the Vienna Atomic Line Database ${ }^{12}$ (VALD, Piskunov et al. 1995; Kupka et al. 1999) in our spectral range of interest. We discarded transitions with excitation potentials (EP) greater than $10 \mathrm{eV}$ or with oscillator strengths $\log g f<-5$.

\footnotetext{
12 http://vald.astro.univie.ac.at/ vald/php/vald.php
}

Table 2

Line List

\begin{tabular}{llcc}
\hline \hline Wavelength $(\AA)^{\mathrm{a}}$ & Species & EP $(\mathrm{eV})$ & $\log g f$ \\
\hline 4100.005 & $\mathrm{Mn} \mathrm{II}$ & 8.129 & -1.184 \\
4100.013 & $\mathrm{NH}$ & 2.202 & -3.421 \\
4100.014 & $\mathrm{NH}$ & 2.387 & -4.433 \\
4100.021 & $\mathrm{NH}$ & 2.335 & -4.289 \\
4100.022 & ${ }^{3} \mathrm{CH}$ & 0.642 & -4.345 \\
4100.048 & $\mathrm{NH}$ & 2.824 & -2.929 \\
4100.051 & $\mathrm{NH}$ & 2.335 & -4.321 \\
4100.055 & $\mathrm{NH}$ & 2.599 & -2.932 \\
4100.084 & $\mathrm{NH}$ & 2.202 & -4.982 \\
4100.088 & $\mathrm{Cr}$ & 4.535 & -1.241 \\
$\ldots$ & $\ldots$ & $\ldots$ & $\ldots$ \\
\hline
\end{tabular}

References. Atomic data come from VALD (Piskunov et al. 1995; Kupka et al. 1999) and NIST (Kramida et al. 2014). CH data come from SCAN (Jorgensen et al. 1996). NH and CN data come from Kurucz (1992).

${ }^{\text {a }}$ Wavelength in air.

(This table is available in its entirety in a machine-readable form.)

We then compiled a separate atomic line list from the National Institute of Standards and Technology (NIST) database $^{13}$ (Kramida et al. 2014). This database is very incomplete, but it generally has more trustworthy, laboratorymeasured atomic transition probabilities than other databases. We replaced entries in the VALD line list with NIST entries where available.

Next, we added molecular transitions. We used the $\mathrm{CN}$ and NH line lists of Kurucz (1992) ${ }^{14}$ and the SCAN line list ${ }^{15}$ for CH from Jorgensen et al. (1996). (Masseron et al. 2014 published a new $\mathrm{CH}$ line list long after we computed the spectral grid and shortly before we submitted this article.) We preserved the isotopic information for each $\mathrm{CH}$ transition so that we could change ${ }^{12} \mathrm{C} /{ }^{13} \mathrm{C}$ in the spectral syntheses.

No line list is perfectly accurate or perfectly complete. We tested our own line list against observed, high-resolution spectra of the Sun and Arcturus (Hinkle et al. 2000). We computed synthetic spectra for both stars using $\mathrm{MOOG}^{16}$ (Sneden 1973) with an updated treatment of electron scattering (Sobeck et al. 2011). For the Sun, we used an ATLAS9 (Kurucz 1993) model atmosphere with $T_{\text {eff }}=5798 \mathrm{~K}$ and $\log g=4.44$. For Arcturus, we used Peterson et al.'s (1993) model atmosphere. Where atomic lines did not match the observed spectra, we changed oscillator strengths. We also introduced a handful of artificial $\mathrm{Fe}_{\mathrm{I}}$ lines to mimic observed lines that did not appear in our original line list. We avoided changing molecular line strengths. Table 2 gives the final line list that we used in our computation of synthetic spectra (Section 3.3.2).

\subsubsection{Spectral Synthesis}

We used MOOG with our existing grid of ATLAS9 model atmospheres (Kirby 2011) to compute synthetic spectra at a range of atmospheric parameters. Table 3 shows the parameters of the spectral grid: the lower and upper ranges and step sizes of wavelength, effective temperature, surface gravity,

\footnotetext{
$13 \mathrm{http}: / /$ nist.gov/pml/data/asd.cfm

14 http://kurucz.harvard.edu/

15 http://astro.ku.dk/ uffegj/

$16 \mathrm{http}: / /$ as.utexas.edu/ chris/moog.html
} 
Table 3

Grid of Synthetic Spectra

\begin{tabular}{lccc}
\hline \hline Parameter & Start & End & Step \\
\hline$\lambda$ & $4100 \AA$ & $4500 \AA$ & $0.02 \AA$ \\
$T_{\text {eff }}$ & $3500 \mathrm{~K}$ & $5600 \mathrm{~K}$ & $100 \mathrm{~K}$ \\
$\ldots$ & $5600 \mathrm{~K}$ & $6400 \mathrm{~K}$ & $200 \mathrm{~K}$ \\
$\log g$ & 0.0 & 4.0 & 0.5 \\
{$[\mathrm{Fe} / \mathrm{H}]$} & -4.0 & 0.0 & 0.1 \\
{$[\alpha / \mathrm{Fe}]$} & -0.8 & +1.2 & 0.1 \\
{$[\mathrm{C} / \mathrm{Fe}]$} & -2.4 & +1.0 & 0.2 \\
$\ldots$ & +1.0 & +3.0 & 0.4 \\
$\ldots$ & +3.0 & +3.5 & 0.5 \\
\hline
\end{tabular}

metallicity, alpha enhancement, and carbon abundance. We used 256 CPUs to compute the 4,835,376 spectra. Although $[\mathrm{C} / \mathrm{Fe}]$ varied in the spectral syntheses, the input model atmospheres were all computed assuming $[\mathrm{C} / \mathrm{Fe}]=0$.

As stars ascend the RGB, they dredge up products of the CNO cycle. We examine this phenomenon in Section 4, but many others have investigated it before. In particular, Keller et al. (2001) showed how the ${ }^{12} \mathrm{C} /{ }^{13} \mathrm{C}$ isotope ratio varies as a function of evolutionary state. The following relation, which we deduced from Keller et al. (2001)'s figure 4, approximates how the ratio changes with decreasing surface gravity:

$$
\begin{aligned}
{ }^{12} \mathrm{C} /{ }^{13} \mathrm{C} & =50 \quad \text { if } \log g>2.7 \\
{ }^{12} \mathrm{C} /{ }^{13} \mathrm{C} & =63 \\
\log g & -120 \text { if } 2.0<\log g \leqslant 2.7 \\
{ }^{12} \mathrm{C} /{ }^{13} \mathrm{C} & =6 \quad \text { if } \log g \leqslant 2.0
\end{aligned}
$$

We used Equation (1) in computing the grid of synthetic spectra.

\subsection{Carbon Abundance Measurements}

We measured carbon abundances by comparing the observed spectra to our new grid. This process involved several steps.

1. Continuum Division: MOOG generates synthetic spectra normalized to the continuum. We divided each observed spectrum by a continuum fit so that it could be compared to the synthetic spectra. We fit the spectrum with a spline with a breakpoint spacing of 200 A. Pixels with fluxes that were greater than 5 standard deviations above or 0.1 standard deviations below the spline were iteratively excluded from the fit so that stellar absorption was not included in the continuum determination. The spectrum was divided by the spline. This continuum was refined in step 3.

2. Preliminary $[\mathrm{C} / \mathrm{Fe}]$ Measurement: We searched the spectral grid for the best-fitting value of $[\mathrm{C} / \mathrm{Fe}]$. The synthetic spectra were smoothed through a Gaussian kernel (2.1 $\AA$ FWHM) to match the observed resolution. We fixed $T_{\text {eff }}, \log g,[\mathrm{Fe} / \mathrm{H}]$, and $[\alpha / \mathrm{Fe}]$ at the values previously determined from the red DEIMOS spectra (see Section 3.2). We used the Levenberg-Marquardt optimization code MPFIT (Markwardt 2012) to find the value of $[\mathrm{C} / \mathrm{Fe}]$ that minimized $\chi^{2}$ in the spectral range $4260-4325 \AA$ (green region in Figures 1 and 3 ), where the spectrum is most sensitive to changes in carbon abundance.
3. Continuum Refinement: The continuum determination in step 1 does not take into account the known variation in flux as a function of wavelength. We refined the continuum by dividing the observed spectrum by the best-fitting synthetic spectrum determined in step 2 . We fit that quotient with a spline with a breakpoint spacing of $150 \AA$. This spacing is slightly finer than in step 1 for a slightly more detailed continuum determination. We iteratively discarded pixels that were above or below the fit by more than one standard deviation.

4. Refined $[\mathrm{C} / \mathrm{Fe}]$ Measurement: We repeated steps 2 and 3 until $[\mathrm{C} / \mathrm{Fe}]$ changed by less than 0.001 between iterations.

In some cases, the spectra allowed only measurements of upper limits. We computed $\chi^{2}$ contours for each star at several values of $[\mathrm{C} / \mathrm{Fe}]$ around the minimum $\chi^{2}$. For stars whose $\chi^{2}$ contours did not rise by at least 1 on both sides of the minimum, we calculated a $2 \sigma$ upper limit. The upper limit was the value of $[\mathrm{C} / \mathrm{Fe}]$ at which $\chi^{2}$ was 4 above the minimum $\chi^{2}$.

Figure 1 shows model spectra of the best-fitting $G$ band spectrum in red. Also shown in light blue are model spectra deviant from the best fit at $[\mathrm{C} / \mathrm{Fe}] \pm 0.2$. Figure 3 shows two pairs of spectra of stars-one pair in Sculptor and one pair in Ursa Minor-with similar luminosity and metallicity but different carbon abundances. The figure demonstrates that the stars measured to have larger $[\mathrm{C} / \mathrm{Fe}]$ have noticeably stronger $\mathrm{G}$ bands.

We also computed the $S_{2}(\mathrm{CH})$ index (Martell et al. 2008a). This index is defined to have minimal sensitivity to nitrogen abundance. It is the ratio of flux in the $\mathrm{G}$ band to flux in two side bands. The blue side band starts at $4212 \AA$. We measured $S_{2}(\mathrm{CH})$ only in spectra with minimum rest wavelengths less than $4212 \AA$. Martell et al. (2008a) defined the band to be measured in units of ADU per pixel. However, the throughput of DEIMOS in the vicinity of the $G$ band diminishes rapidly toward the blue. Consequently, we measured $S_{2}(\mathrm{CH})$ in continuum-corrected flux rather than the original ADU per pixel.

Table 4 gives the $[\mathrm{C} / \mathrm{Fe}]$ and $S_{2}(\mathrm{CH})$ measurements for our $\mathrm{GC}$ and $\mathrm{dSph}$ sample. The last column identifies the 11 carbon stars for which we were unable to measure $[\mathrm{Fe} / \mathrm{H}]$ or $[\mathrm{C} / \mathrm{Fe}]$. The table also gives photometric information, including the absolute luminosity for each star relative to the solar luminosity. We used bolometric corrections based on theoretical isochrones (Demarque et al. 2004; Girardi et al. 2004) and previously measured distance moduli to compute the luminosities: 19.83 for NGC 2419 (Harris et al. 1997), 15.21 for M68 (McClure et al. 1987), 15.08 for M15 (Durrell \& Harris 1993), 19.67 for Sculptor (Pietrzyński et al. 2008), 20.72 for Fornax (Rizzi et al. 2007), 19.18 for Ursa Minor (Mighell \& Burke 1999), and 19.84 for Draco (Bellazzini et al. 2002). The table also includes $T_{\text {eff }}, \log g,[\alpha / \mathrm{Fe}]$, and $\mathrm{S} / \mathrm{N}$, calculated as the median absolute deviation of the observed spectrum from the best-fitting spectrum in the range 4250-4350 A.

\subsubsection{Estimation of Uncertainties}

Although MPFIT provides a covariance matrix for each minimization, this estimate of uncertainty on $[\mathrm{C} / \mathrm{Fe}]$ does not account for uncertainty in the placement of the continuum. Instead of using the covariance matrix, we calculated Monte 


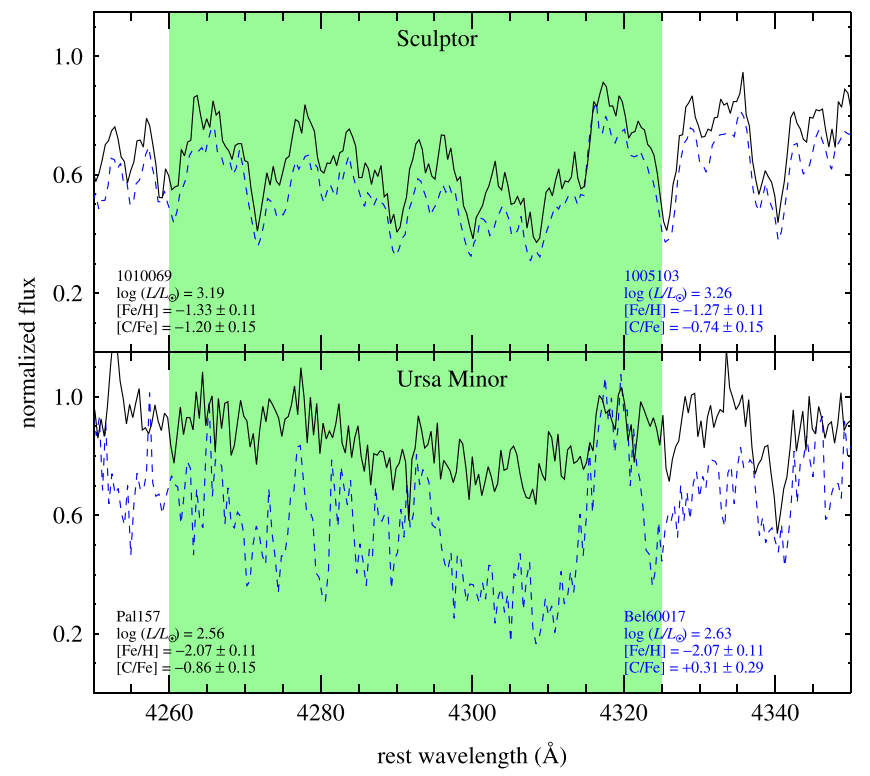

Figure 3. $G$ band spectra of pairs of stars with similar luminosity, similar metallicity, and differing carbon abundance in Sculptor (top) and Ursa Minor (bottom). The stellar parameters listed on the left (right) of each panel correspond to the solid black (dashed blue) spectrum. As in Figure 1, only the green shaded region is used in the measurement of $[\mathrm{C} / \mathrm{Fe}]$.

Carlo uncertainties on $[\mathrm{C} / \mathrm{Fe}]$ by resampling the observed spectrum.

After we determined the best-fitting carbon abundance, we added noise to the spectrum after it was continuum-normalized according to step 1 in Section 3.4. The new flux in each noiseadded pixel was the original flux plus a value drawn from a Gaussian random distribution with a standard deviation equal to spec2d's estimate of the flux error in that pixel. We repeated steps 2-4 above with the noise-added spectrum.

We repeated this process for a total of 1000 noise realizations. We took the base uncertainty on $[\mathrm{C} / \mathrm{Fe}]$ as the standard deviation of all 1000 trials. Although this procedure accounts for random uncertainty, it does not include all sources of systematic error. We added $0.1 \mathrm{dex}$ as an estimate of systematic error in quadrature with the Monte Carlo uncertainty for the final uncertainty on $[\mathrm{C} / \mathrm{Fe}]$. This systematic uncertainty is approximately the value that is required to account for the rms of the distribution of our measurements of $[\mathrm{C} / \mathrm{Fe}]$ versus literature measurements (Figure 5, discussed in Section 3.4.3).

We computed errors on $S_{2}(\mathrm{CH})$ from the same noise-added spectra. We did not add any systematic error to these measurements.

\subsubsection{Effect of Nitrogen and Oxygen}

The measurement of carbon could be affected by the abundances of other elements. First, other elements that form diatoms with carbon affect the molecular equilibrium, changing the amount of carbon available to form $\mathrm{CH}$. Second, some elements contribute to the opacity in the stellar atmosphere. Changes in their abundances can affect the structure of the atmosphere and the strength of the $\mathrm{G}$ band. The most important elements to consider for these effects are nitrogen and oxygen because they are abundant, and they form diatoms with carbon. Unfortunately, we can measure neither element directly from our spectra. However, we can quantify their possible effects on our carbon abundance measurements.
We explored the effects of molecular equilibrium between $\mathrm{CH}$ and $\mathrm{CN}$ by making syntheses with MOOG with varying abundances of $\mathrm{N}$. We took three stars in M15 $([\mathrm{Fe} / \mathrm{H}] \approx-2.4)$ as test cases: 13701, which is below the RGB luminosity function bump; 43026, which is at the top of the RGB; and 47866, which is carbon-enhanced (see Section 4). We also tested two stars in Fornax to explore more metal-rich $([\mathrm{Fe} / \mathrm{H}] \approx-1)$ stars: 52752 at $\log \left(L / L_{\odot}\right)=2.0$ and 40828 at the top of the RGB. We synthesized spectra with $[\mathrm{N} / \mathrm{Fe}]$ values of $-2.0,-1.0,-0.5,-0.2,0.0,+0.2,+0.5,+1.0$, and +2.0 . In no case did the flux of any pixel in the $\mathrm{G}$ band change by more than $1 \%$.

Our carbon abundance measurements already account for varying elemental abundances that might affect the structure of the stellar atmospheres. We previously measured four $\alpha$ elements $(\mathrm{Mg}, \mathrm{Si}, \mathrm{Ca}$, and $\mathrm{Ti}$ ) from red DEIMOS spectra (Kirby et al. 2010). The stellar atmospheres we used to measure carbon abundances reflect those $[\alpha / \mathrm{Fe}]$ ratios. VandenBerg et al. (2012) showed that at temperatures found in the atmospheres of cool giants, $\mathrm{Mg}$ and $\mathrm{Si}$ are the most important metals for determining the opacity. As a result, changing $[\alpha / \mathrm{Fe}]$ (including $[\mathrm{Mg} / \mathrm{Fe}]$ and $[\mathrm{Si} / \mathrm{Fe}]$ ) can alter the atmospheric structure and therefore affect the measurements of all elemental abundances.

We recomputed all of the $[\mathrm{C} / \mathrm{Fe}]$ measurements assuming $[\alpha / \mathrm{Fe}]=0.0$ rather than the values previously determined by Kirby et al. (2010). Here, $\alpha$ refers to O, Ne, Mg, Si, S, Ar, Ca, and Ti. This value of $[\alpha / \mathrm{Fe}]$ is used to compute both the model atmosphere and the spectral synthesis. Hence, the syntheses include the effects of the varying atmospheric structure and the changing molecular equilibrium between $\mathrm{CH}$ and $\mathrm{CO}$. Figure 4 shows the response of $[\mathrm{C} / \mathrm{Fe}]$ to the change in $[\alpha / \mathrm{Fe}]$. Changing $[\alpha / \mathrm{Fe}]$ by $\pm 0.6 \mathrm{dex}$ - much more than the mean uncertainty in $[\alpha / \mathrm{Fe}], 0.2 \mathrm{dex}$-results in a change of approximately $\pm 0.1 \mathrm{dex}$ in $[\mathrm{C} / \mathrm{Fe}]$. Forcing $[\alpha / \mathrm{Fe}]$ to be 0.0 results in $|\Delta[\mathrm{C} / \mathrm{Fe}]|<0.1$ dex for $94 \%$ of the $\mathrm{dSph}$ stars and $|\Delta[\mathrm{C} / \mathrm{Fe}]|<0.05$ dex for $75 \%$ of the dSph stars. We conclude that uncertainty in $[\alpha / \mathrm{Fe}]$ is not a major source of uncertainty in our carbon abundance measurements.

In conclusion, uncertainties in nitrogen, oxygen, and other $\alpha$ element abundances are minor contributors to the error budget of our $[\mathrm{C} / \mathrm{Fe}]$ measurements.

\subsubsection{Validation}

We compared our low-resolution measurements of $[\mathrm{C} / \mathrm{Fe}]$ with previous high- and low-resolution measurements of $[\mathrm{C} / \mathrm{Fe}]$ for the same stars. We found overlapping stars with highresolution measurements in the literature for NGC 2419 (Cohen \& Kirby 2012), M68 (Venn et al. 2012), Draco (Fulbright et al. 2004; Cohen \& Huang 2009), and Ursa Minor (Cohen \& Huang 2010). Shetrone et al. (2013) also published measurements of $[\mathrm{C} / \mathrm{Fe}]$ in Draco based on low-resolution Keck/LRIS spectroscopy. All of these measurements are also based on the $\mathrm{G}$ band.

The left panel of Figure 5 shows the comparison between $[\mathrm{C} / \mathrm{H}]$ measured from DEIMOS and $[\mathrm{C} / \mathrm{H}]$ as published in the aforementioned references. The right panel shows $[\mathrm{C} / \mathrm{Fe}]$. We shifted all abundances to our adopted solar abundance scale ${ }^{17}$ : $A(\mathrm{C})=8.56$ (Anders \& Grevesse 1989) and $A(\mathrm{Fe})=7.52$

\footnotetext{
${ }^{17} A(\mathrm{X})=12+\log (n(\mathrm{X}) / n(\mathrm{H}))$ where $n(\mathrm{X})$ is the number density of element $\mathrm{X}$.
} 
Table 4

Carbon Abundances

\begin{tabular}{|c|c|c|c|c|c|c|c|c|c|c|c|c|c|}
\hline System & Name & $\begin{array}{c}\text { R.A. } \\
\text { (J2000) }\end{array}$ & $\begin{array}{c}\text { Decl. } \\
(\mathrm{J} 2000)\end{array}$ & $\begin{array}{l}\log L \\
\left(L_{\odot}\right)\end{array}$ & $\begin{array}{l}\mathrm{S} / \mathrm{N} \\
\left(\AA^{-1}\right)\end{array}$ & $\begin{array}{l}T_{\text {eff }} \\
(\mathrm{K})\end{array}$ & $\begin{array}{c}\log g \\
\left(\mathrm{~cm} \mathrm{~s}^{-2}\right)\end{array}$ & $\begin{array}{l}\mathrm{Fe} / \mathrm{H}] \\
(\mathrm{dex})\end{array}$ & $\begin{array}{l}{[\alpha / \mathrm{Fe}]} \\
(\operatorname{dex})\end{array}$ & $\begin{array}{l}\bar{C} / \mathrm{Fe}] \\
(\mathrm{dex})\end{array}$ & $\begin{array}{c}\mathrm{C} / \mathrm{Fe}]_{\text {corr }}{ }^{\mathrm{a}} \\
(\mathrm{dex})\end{array}$ & $\begin{array}{c}S_{2}(\mathrm{CH}) \\
(\mathrm{mag})\end{array}$ & $\overline{\text { C star? }}$ \\
\hline Ursa Minor & Bel60031 & 150738.73 & +671356.4 & 2.94 & $\ldots$ & $\ldots$ & $\ldots$ & $\ldots$ & $\ldots$ & $\ldots$ & $\ldots$ & $\ldots$ & $\mathrm{C}$ \\
\hline Ursa Minor & Bel60021 & 150750.88 & +671112.9 & 2.63 & 99 & 4495 & 1.30 & $-2.43 \pm 0.11$ & $+0.08 \pm 0.15$ & $-0.65 \pm 0.16$ & -0.01 & $\ldots$ & $\ldots$ \\
\hline Ursa Minor & Bel60469 & 150755.52 & +671130.2 & 1.74 & 32 & 4772 & 2.33 & $-2.78 \pm 0.25$ & $\ldots$ & $\leqslant+0.44$ & $\leqslant+0.45$ & $1.75 \pm 0.33$ & $\ldots$ \\
\hline Ursa Minor & Bel60230 & 150759.77 & +671528.8 & 2.28 & $\cdots$ & $\ldots$ & $\ldots$ & $\ldots$ & $\ldots$ & $\ldots$ & $\ldots$ & $\ldots$ & $\mathrm{C}$ \\
\hline Ursa Minor & Bel60123 & 150759.77 & +671141.4 & 2.10 & 56 & 4826 & 1.96 & $-2.36 \pm 0.13$ & $+0.35 \pm 0.19$ & $\leqslant+0.06$ & $\leqslant+0.13$ & $1.60 \pm 0.11$ & $\cdots$ \\
\hline Ursa Minor & Bel70584 & 150808.20 & +670643.8 & 1.62 & 24 & 4899 & 2.49 & $-2.30 \pm 0.21$ & $-0.19 \pm 0.68$ & $\leqslant+0.55$ & $\leqslant+0.56$ & $\ldots$ & $\cdots$ \\
\hline Ursa Minor & Bel70182 & 150808.29 & +6704 41.0 & 2.00 & 25 & 4803 & 2.06 & $-2.02 \pm 0.16$ & $-0.17 \pm 0.46$ & $\leqslant+0.90$ & $\leqslant+0.94$ & $\ldots$ & $\ldots$ \\
\hline Ursa Minor & Bel60017 & 150809.06 & +670921.6 & 2.63 & 70 & 4526 & 1.33 & $-2.07 \pm 0.11$ & $+0.17 \pm 0.13$ & $+0.31 \pm 0.27$ & +0.73 & $2.13 \pm 0.05$ & $\ldots$ \\
\hline Ursa Minor & Bel50017 & 150810.51 & +671707.1 & 2.60 & 111 & 4529 & 1.35 & $-2.18 \pm 0.11$ & $+0.04 \pm 0.08$ & $-0.70 \pm 0.21$ & -0.10 & $1.59 \pm 0.05$ & $\cdots$ \\
\hline Ursa Minor & Bel60819 & 150812.07 & +671015.5 & 1.27 & 13 & 5198 & 2.93 & $-1.85 \pm 0.33$ & $\ldots$ & $\leqslant-0.26$ & $\leqslant-0.25$ & $1.54 \pm 0.17$ & $\ldots$ \\
\hline$\cdots$ & $\ldots$ & $\ldots$ & $\ldots$ & $\ldots$ & $\cdots$ & $\ldots$ & $\ldots$ & $\ldots$ & $\cdots$ & $\cdots$ & $\cdots$ & $\ldots$ & $\cdots$ \\
\hline
\end{tabular}

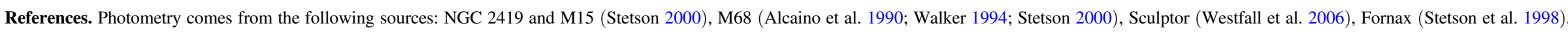
Ursa Minor (Bellazzini et al. 2002), Draco (Ségall et al. 2007).

${ }^{\mathrm{a}}[\mathrm{C} / \mathrm{Fe}]$ corrected for stellar evolutionary state according to Placco et al. (2014).

(This table is available in its entirety in a machine-readable form.) 


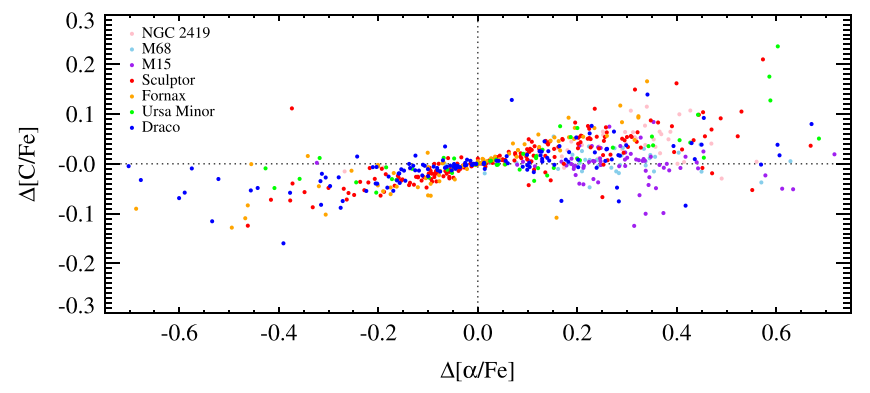

Figure 4. The response of $[\mathrm{C} / \mathrm{Fe}]$ to changing $[\alpha / \mathrm{Fe}]$ in the stellar atmosphere. The $x$-axis shows the difference between the $[\alpha / \mathrm{Fe}]$ values used in our fiducial measurements of $[\mathrm{C} / \mathrm{Fe}]$ and $[\alpha / \mathrm{Fe}]=0.0$.

(Sneden et al. 1992). The deviations reach up to 0.8 dex. The deviations are also about the same for $[\mathrm{C} / \mathrm{Fe}]$ as they are for $[\mathrm{C} / \mathrm{H}]$, which indicates that differences in atmospheric parameters like $T_{\text {eff }}$ are not the reason for the discrepancy. On the other hand, the degree of deviation depends largely on the source of the measurement. For example, our $[\mathrm{C} / \mathrm{H}]$ measurements are $0.3-0.4$ dex above Venn et al.'s $[\mathrm{C} / \mathrm{H}]$ measurements from VLT/FLAMES, but our measurements are on average $\sim 0.4$ dex below Cohen \& Huang's, Cohen \& Kirby's, and Fulbright et al.'s measurements from Keck/HIRES. Our measurements largely agree with Shetrone et al.'s Keck/LRIS measurements.

The origin of the discrepancies might be spectral resolution or the details of the carbon abundance measurements. At first glance, the spectral resolution is suspect because our low-

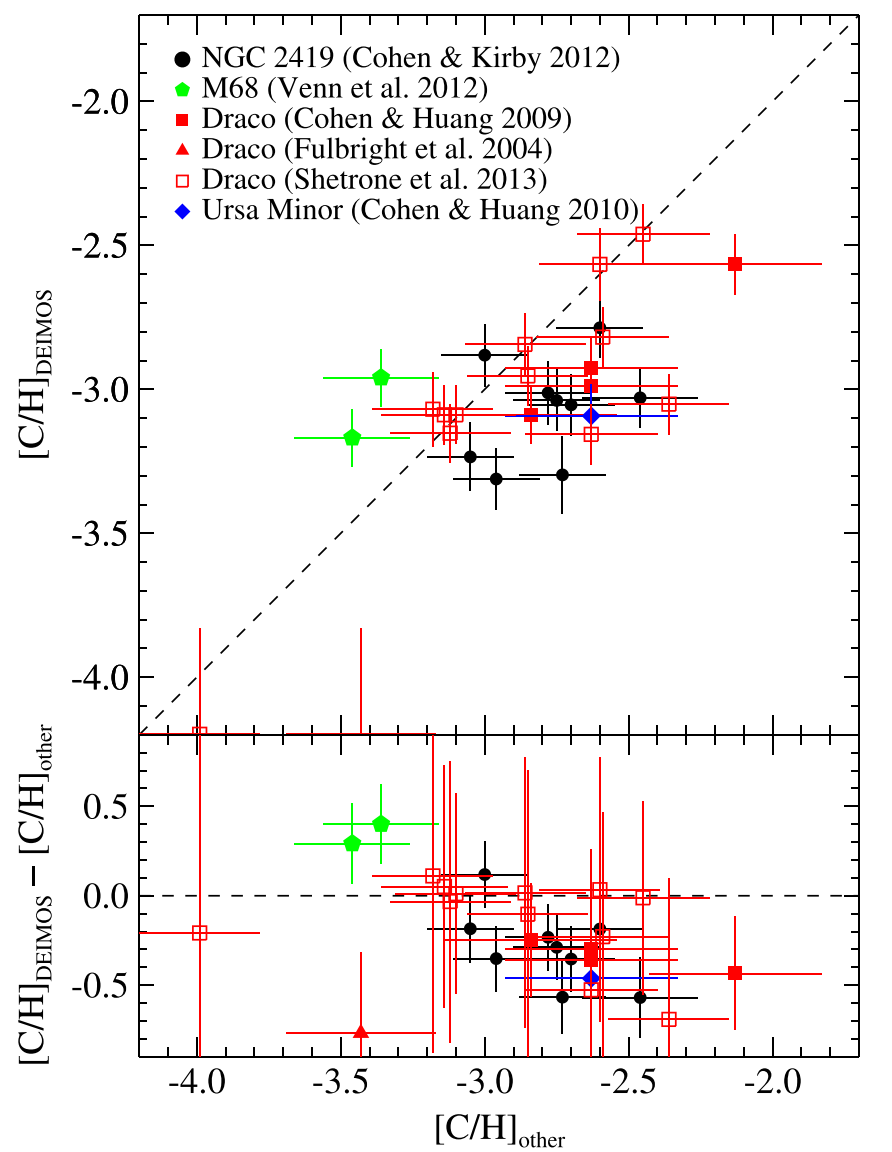

resolution measurements agree most with another low-resolution study (Shetrone et al. 2013). However, our measurements disagree with Venn et al.'s (2012) in the opposite direction from the other two high-resolution studies. Therefore, we investigated the possibility that differences in the line list caused the offset. We compared our line list (Table 2) with J. Cohen's line list. Between 4274 and $4232 \AA$, our list contains 2.5 times more $\mathrm{CH}$ lines. The higher density of lines makes the $\mathrm{G}$ band stronger. Hence, for a fixed observed $\mathrm{G}$ band strength, we would measure a lower carbon abundance with our line list than with Cohen's line list. Our line list has a similar density of lines to Shetrone et al.'s list. Therefore, it is plausible that a different line list is the main source of disagreement of carbon abundance measurements from the $\mathrm{G}$ band.

\section{ASTRATION OF CARBON ON THE UPPER RGB}

The destruction of carbon on the RGB above the luminosity function bump is well documented (e.g., Suntzeff 1981; Carbon et al. 1982; Smith \& Briley 2006). As previous investigators have done, we searched for signatures of carbon astration in GCs. GCs are the easiest stellar systems to isolate stellar luminosity as an independent variable because the cluster stars have similar ages, metallicities, and distances.

Figure 6 shows the trend of carbon enhancement $([\mathrm{C} / \mathrm{Fe}])$ with luminosity. The carbon abundance remains flat until giants reach $\log \left(L / L_{\odot}\right)=1.6$, whereupon $[\mathrm{C} / \mathrm{Fe}]$ declines, presumably due to mixing that begins at the luminosity function bump. We fit a line to the combined trend of $[\mathrm{C} / \mathrm{Fe}]$ with $[\mathrm{Fe} / \mathrm{H}]$ for all three GCs, fixing the luminosity of the bump at

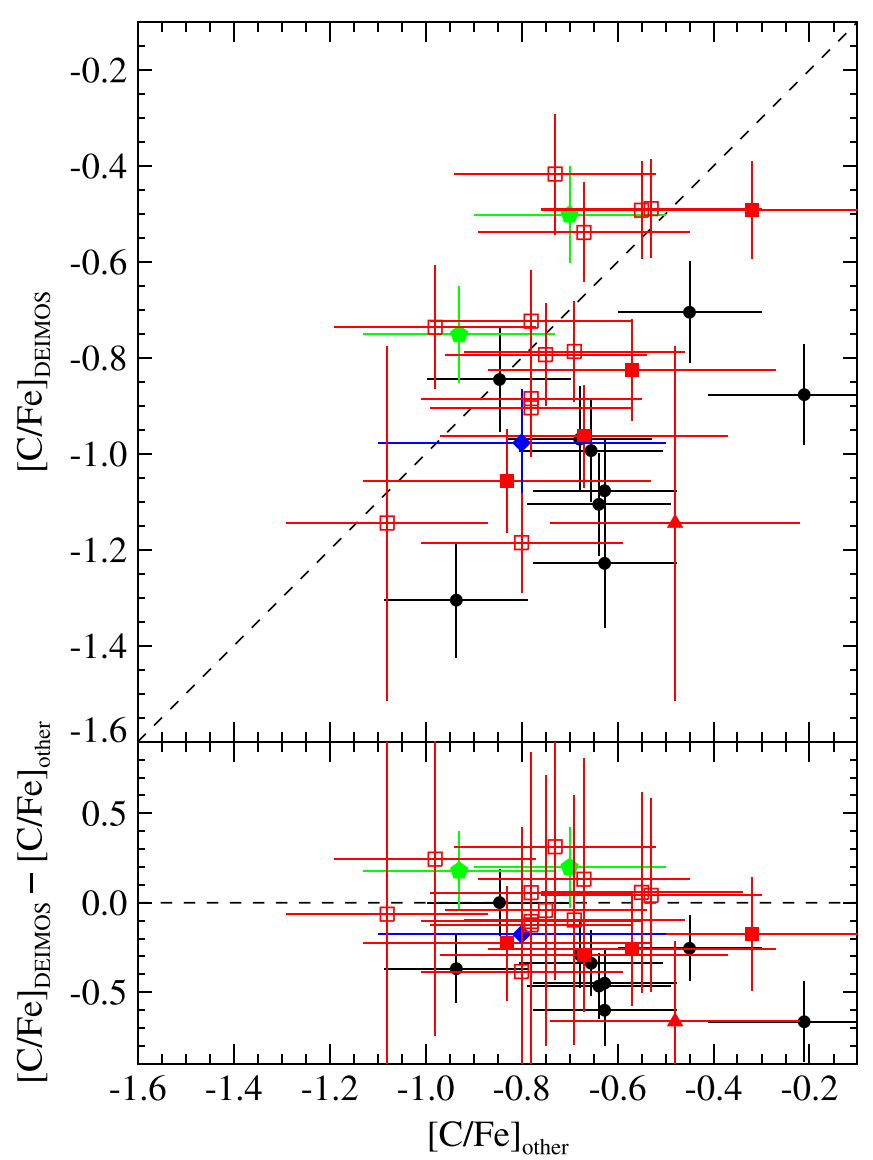

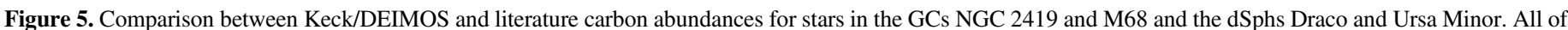
the literature measurements come from Keck/HIRES except for the studies of Venn et al. (2012, Magellan/MIKE) and Shetrone et al. (2001, Keck/LRIS). 


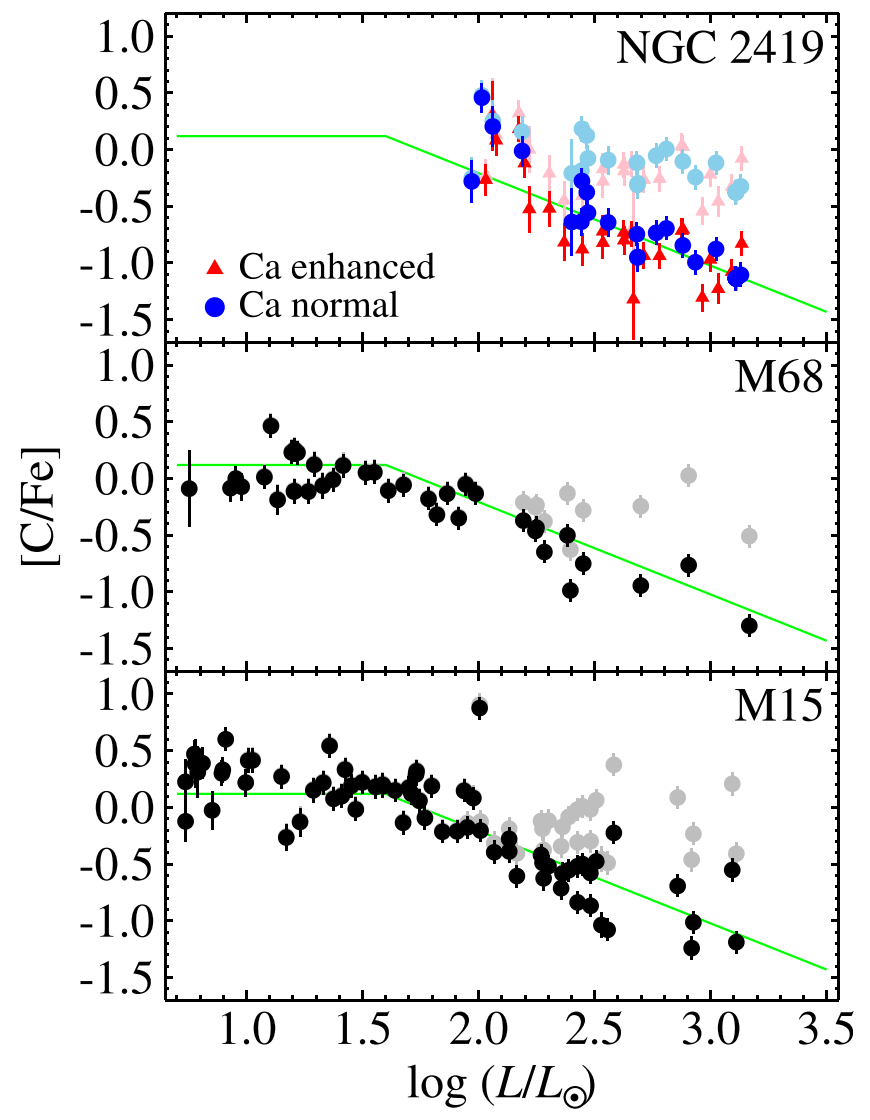

Figure 6. The decrease in carbon enhancement with red giant luminosity in two globular clusters. The giants in NGC 2419 are separated into calcium-enhanced (red triangles) and normal-calcium groups (red circles). The green lines illustrate of the destruction of carbon as photospheric material is processed by the CNO cycle on the upper RGB (Equation (2)). The faded points show the $[\mathrm{C} / \mathrm{Fe}]$ measurements corrected for the destruction of carbon with increasing luminosity according to the calculations of Placco et al. (2014).

$\log \left(L / L_{\odot}\right)=1.6$. The best fit line, represented in green in Figure 6, is

$$
\begin{array}{ll}
{[\mathrm{C} / \mathrm{Fe}]=0.12 \pm 0.06} & \text { if } \log \left(L / L_{\odot}\right) \leqslant 1.6 \\
{[\mathrm{C} / \mathrm{Fe}]=(1.42 \pm 0.06)} & \\
\quad-(0.82 \pm 0.02) \log \left(L / L_{\odot}\right) & \text { if } \log \left(L / L_{\odot}\right)>1.6
\end{array}
$$

The amount of carbon astration in GCs could be computed from this empirical trend, but we also considered theoretical estimates of the amount of carbon depletion. Placco et al. (2014) calculated corrections based on models of mixing on the upper RGB. The corrections depend on surface gravity, metallicity, and uncorrected carbon abundance. ${ }^{18}$ We used their models to compute a correction for each star in our sample. Figure 6 shows in faded colors Placco et al.'s corrections applied to the GC sample. The faded points have less dependence on luminosity than the uncorrected points. Although the empirical linear fit to the data (Equation (2)) is a better fit than Placco et al.'s models, the models are ab initio

\footnotetext{
18 The correction depends slightly on $[\mathrm{N} / \mathrm{Fe}]$. We assumed $[\mathrm{N} / \mathrm{Fe}]=0$. Gratton et al. (2000) showed that $[\mathrm{N} / \mathrm{Fe}]$ climbs to about 0.5 at the tip of the RGB in metal-poor field stars. Placco et al.'s (2014) corrections change by $<0.05 \mathrm{dex}$ for changes in $[\mathrm{N} / \mathrm{Fe}]$ of $0.5 \mathrm{dex}$. For the range of stellar parameters in our sample, the difference from the $[\mathrm{N} / \mathrm{Fe}]=0$ correction exceeds 0.1 dex only for $[\mathrm{N} / \mathrm{Fe}] \geqslant+1$, a condition that very few, if any, of the stars in our sample would satisfy.
}

predictions. They can be applied to data in environments that are not as tightly controlled as GCs. We use the Placco et al. (2014) corrections for dSph stars in Section 5.

Martell et al. (2008c) found that the rate of carbon depletion as giants evolve depends on metallicity, which is consistent with the thermohaline mixing mechanism for the change in elemental composition with luminosity (Charbonnel \& Zahn 2007; Charbonnel \& Lagarde 2010). Unfortunately, we cannot test for metallicity dependence because the GCs we observed have similar metallicities. According to Harris (1996), the metallicities are $[\mathrm{Fe} / \mathrm{H}]=-2.15$ (NGC 2419, Suntzeff et al. 1988), -2.23 (M68, Lee et al. 2005), and -2.37 (M15, Takeda et al. 2009). Martell et al. found carbon depletion rates of $d[\mathrm{C} / \mathrm{Fe}] / d \log \left(L / L_{\odot}\right)=-0.7$ to -1.5 above the luminosity function bump for the metallicity range of these three GCs. Our value of $-0.82 \pm 0.02$ (Equation (2)) falls in this range.

GCs have primordial abundance variations (Gratton et al. 2004), including carbon (e.g., Cohen 1999). The dispersion in $[\mathrm{C} / \mathrm{Fe}]$ at fixed luminosity can give a sense of the differential enrichment in carbon between the first and subsequent generations of star formation. We calculated this dispersion by finding the residuals between $[\mathrm{C} / \mathrm{Fe}]$ and the kinked linear trend. The trend is similar to Equation (2), but we fit each GC individually to calculate the dispersion. We then normalized the residuals by the measurement uncertainty: $\Delta=([\mathrm{C} / \mathrm{Fe}]-[\mathrm{C} / \mathrm{Fe}]($ trend $)) / \delta[\mathrm{C} / \mathrm{Fe}]$. If there is no intrinsic scatter in $[\mathrm{C} / \mathrm{Fe}]$ beyond the measurement uncertainty, then the standard deviation of $\Delta$ should be 1 . Instead, we found 1.6 for NGC 2419, 1.5 for M68, and 2.1 for M15. (We excluded star 47866, discussed below, from M15.) This measurement of dispersion demands that we estimated measurement uncertainties accurately. If we underestimated the uncertainties, then the standard deviation of $\Delta$ will appear erroneously larger than 1 . Taken at face value, we have found evidence for intrinsic scatter in $[\mathrm{C} / \mathrm{Fe}]$. We see no evidence for bimodality in $[\mathrm{C} / \mathrm{Fe}]$, but the scatter is not much larger than the uncertainties. Nonetheless, CN bands do show bimodality in some GCs (Smith \& Briley 2005, 2006; Martell et al. 2008b), reflecting that primordial $\mathrm{C}$ and $\mathrm{N}$ abundances in clusters were bimodal, independent of the present-day astration on the upper RGB. This bimodality can even be found on the main sequence of some clusters (e.g., 47 Tucanae, Harbeck et al. 2003).

Cohen et al. (2011), Cohen \& Kirby (2012), and Mucciarelli et al. (2012) discovered that NGC 2419 has an extremely unusual chemical composition. The $\mathrm{Mg}$ abundances vary from star to star by a factor of 25 , and the variation is anticorrelated with abundances of other elements, including $\mathrm{K}$ and $\mathrm{Ca}$. In a sample of 13 red giants with high-resolution spectra, Cohen \& Kirby (2012) did not find any correlation between C and any of the elements with an intracluster dispersion. We looked in our larger sample for such a correlation by separating the NGC 2419 stars into Ca-enhanced and Ca-normal. We estimated $\mathrm{Ca}$ enhancement by measuring the equivalent widths (EWs) of the near-infrared Ca triplet. We converted these EWs to metallicity estimates using Starkenburg et al.'s (2010) formula. The stars with $[\mathrm{Fe} / \mathrm{H}]_{\mathrm{CaT}}<-2.2$ (Ca-normal) are represented by blue circles in Figure 6. Stars with $[\mathrm{Fe} / \mathrm{H}]_{\mathrm{CaT}} \geqslant-2.2$ (Caenhanced) are red triangles. There is no noticeable difference between the two populations. Hence, we confirm that carbon did not participate in the unusual nucleosynthesis that affected heavier elements in NGC 2419. 
Figure 7 shows an alternate way to display the destruction of carbon as red giants ascend the RGB. The CMD color-codes the stars, with cooler colors corresponding to lower $[\mathrm{C} / \mathrm{Fe}]$. In general, the plotting color is cooler at the top of the RGB compared to less luminous stars. In M68 and M15, which are the GCs observed to sufficient depth, most of the stars below the luminosity function bump $\left(I_{0} \sim 14\right)$ are colored green. This color corresponds to the constant pre-bump value of $[\mathrm{C} / \mathrm{Fe}]=+0.12$.

Note the one carbon-rich star in M15, which is also visible in Figure 6. Star 47866, a confirmed radial velocity member of M15, stands out as lone orange point $([\mathrm{C} / \mathrm{Fe}]=+0.88 \pm 0.10)$ in Figure 7 in a sea of dark green points $([\mathrm{C} / \mathrm{Fe}] \sim-0.2)$. The carbon abundance is high enough that the near-infrared spectrum shows some $\mathrm{CN}$ absorption (Figure 2). This star was also previously identified by J. Cohen to be carbon-rich from an unpublished Keck/HIRES spectrum. That spectrum shows a weak $\mathrm{C}_{2}$ bandhead at $5163 \AA$ in addition to a very strong G band. Also note that this star lies slightly blueward of the main RGB locus. The CMD position indicates that star 47866 is an AGB star.

Carbon-enhanced stars in GCs are rare. Cohen et al. (1978) and Mouhcine (2002) explain that C stars appear after several hundred million years, long after the end of star formation in a GC. None of the GC stars would have been pre-enriched by carbon from an AGB star. Additionally, the lowest-mass AGB star that produces copious amounts of carbon is about $2 M_{\odot}$. Such a star would live for about $1 \mathrm{Gyr}$. Hence, all carbonproducing AGB stars in GCs should have died long ago.

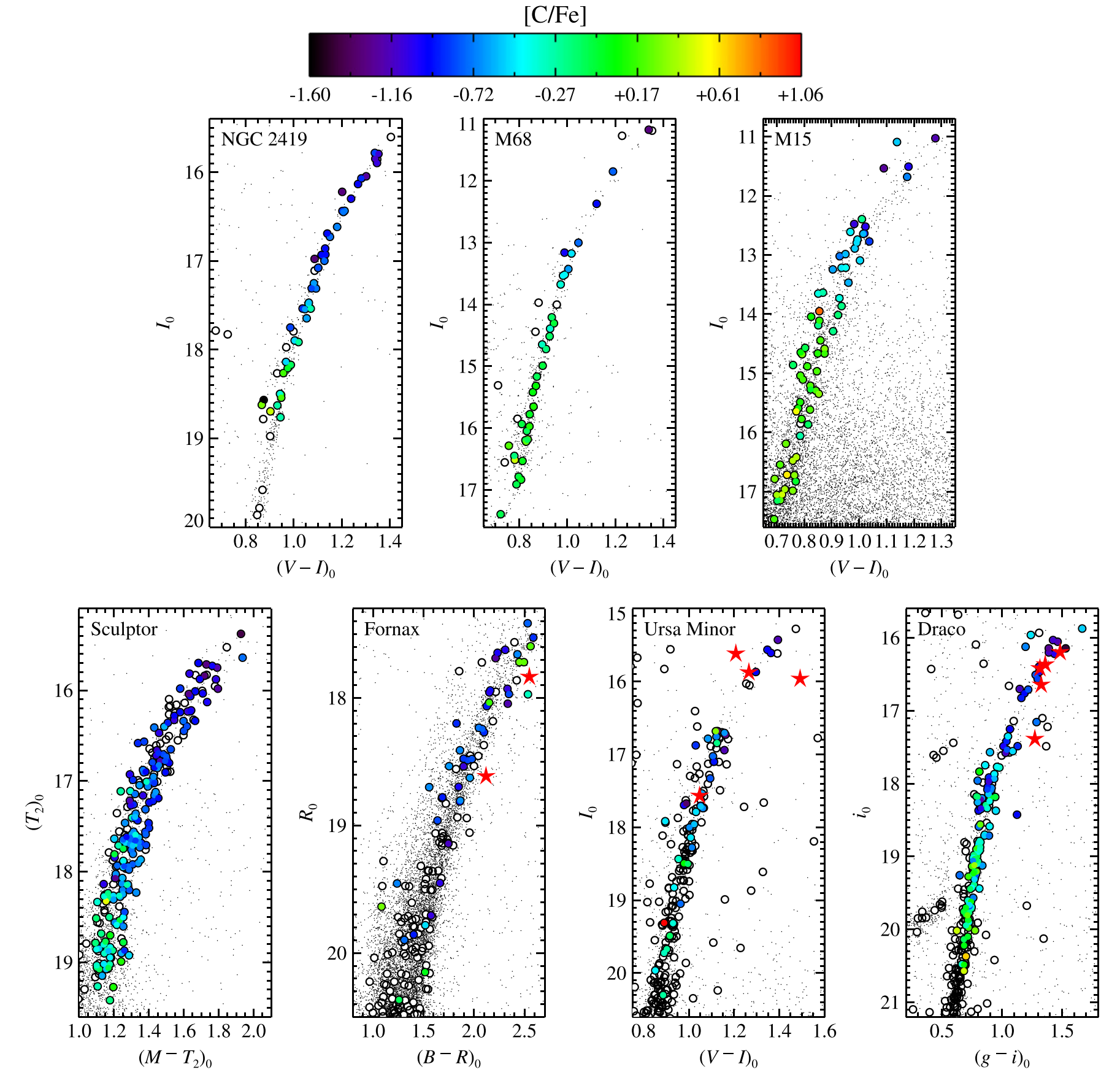

$[\mathrm{C} / \mathrm{Fe}]$

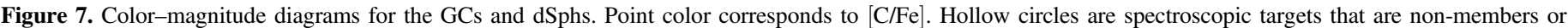
spectra where $[\mathrm{C} / \mathrm{Fe}]$ was unable to be measured. Red, five-pointed symbols indicate very carbon rich stars for which we did not attempt to measure $[\mathrm{C} / \mathrm{Fe}]$. 
Nonetheless, there are a few examples of C-rich stars in GCs. The GC $\omega$ Centauri hosts at least seven known C stars (Harding 1962; Dickens 1972; Bond 1975; Cowley \& Crampton 1985; van Loon et al. 2007). However, $\omega$ Centauri is known to have an unusually long star formation history (SFH) for a GC (Hilker et al. 2004; Villanova et al. 2007). Regardless, C stars can be found in even more ordinary GCs, like M14 (Côté et al. 1997), Lynga 7 (Matsunaga 2006; Feast et al. 2013), and NGC 6426 (Sharina et al. 2012).

$\mathrm{C}$ stars can be formed as the result of mass transfer from an AGB star. In most cases, the AGB star has now evolved into a white dwarf. There is photometric (Böhm-Vitense et al. 2000) and spectroscopic (Lucatello et al. 2005; Starkenburg et al. 2014) evidence that most or all C stars-especially those enhanced in $s$-process elements-have binary companions. Binarity is often diagnosed through radial velocity variability over multiple measurements. Although we observed 47866 twice, the observations were separated by only $24 \mathrm{hr}$. We found no evidence for binarity from this very short time baseline. An alternative possibility is that the existing C-rich AGB star formed in a stellar merger, the result of which would be massive enough to generate carbon in its AGB phase (e.g., Feast et al. 2013). Stellar mergers are a plausible origin for blue stragglers in GCs (Mateo et al. 1990; Sills et al. 2013), so it is conceivable that this carbon-rich star is also the product of a merger.

\section{CARBON IN DWARF SPHEROIDAL GALAXIES}

Unlike GCs, dSphs contain stars with a range of ages and metallicities. The more complex stellar populations complicate the evolution of carbon. First, dSph stars need not start their lives with the same $\mathrm{C}$ abundance. $\mathrm{GC}$ stars were formed from well-mixed gas polluted only by SNe II and AGB stars in a limited mass range. Figure 6 and Equation (2) show that all of the pre-bump stars within a GC have roughly the same value of $[\mathrm{C} / \mathrm{Fe}] \sim+0.12$. On the other hand, the progenitors of $\mathrm{dSph}$ stars can have a range of metallicities. They can also form from inhomogeneously mixed gas polluted by $\mathrm{SNe}$ of various types and AGB stars of various masses and metallicities. Second, the star formation duration of dSphs is long enough for 2-5 $M_{\odot}$ AGB stars-the ones that generate copious amount of carbon-to pollute the interstellar medium while the galaxy is still forming stars. As a result, AGB stars influence the evolution of carbon differently in dSphs than in GCs. Third, the stellar density of dSphs is significantly lower than GCs, which reduces the stellar merger rate. Consequently, the merger formation channel for prematurely C-rich stars (possibly like 47866 in M15) is suppressed.

The bottom panels of Figure 7 show that the decline of $[\mathrm{C} / \mathrm{Fe}]$ with increasing stellar luminosity is just as present in $\mathrm{dSphs}$ as in GCs. However, there is a larger spread in $[\mathrm{C} / \mathrm{Fe}]$ at fixed luminosity in the dSphs. Figure 8 shows the astration of carbon in dSphs, just as Figure 6 shows the same for GCs. The green lines in Figure 8 are the same as in Figure 6. They were not re-fit to the dSph data. The slope of the line roughly tracks the decline of $[\mathrm{C} / \mathrm{Fe}]$ in the dSphs, especially in Sculptor and Draco.

The scatter in $[\mathrm{C} / \mathrm{Fe}]$ at fixed luminosity exceeds the scatter expected from measurement uncertainty. The residuals are correlated with $[\mathrm{Fe} / \mathrm{H}]$, which is represented by the color coding in Figure 8. The residual metallicity trend is especially apparent in Ursa Minor and Draco, the most metal-poor of the four
dSphs we observed. The stars with the lowest $[\mathrm{Fe} / \mathrm{H}]$ have the highest $[\mathrm{C} / \mathrm{Fe}]$ at fixed luminosity. This trend indicates that $\mathrm{C}$ abundance did not increase as quickly as Fe abundance as star formation progressed in the dSphs. Later forming stars, which have higher $[\mathrm{Fe} / \mathrm{H}]$, started with lower $[\mathrm{C} / \mathrm{Fe}]$ ratios than their predecessors.

Figure 9 shows the trend in another way. The $x$-axis is $[\mathrm{Fe} / \mathrm{H}]$ instead of luminosity. Instead of $[\mathrm{C} / \mathrm{Fe}]$, we plot $[\mathrm{C} / \mathrm{Fe}]_{\text {corr }}$, which is corrected for the depletion due to astration on the upper RGB according to Placco et al. (2014). This correction removes the luminosity dependence so that we may isolate $[\mathrm{Fe} / \mathrm{H}]$ as the independent variable. Figure 9 confirms that $[\mathrm{C} / \mathrm{Fe}]_{\text {corr }}$ declines with increasing $[\mathrm{Fe} / \mathrm{H}]$ in Draco. The trend is also apparent in Ursa Minor and Sculptor, where stars with $[\mathrm{Fe} / \mathrm{H}]<-2.2$ have higher $[\mathrm{C} / \mathrm{Fe}]_{\text {corr }}$ on average than stars with $[\mathrm{Fe} / \mathrm{H}]>-2.2$. The sample of stars in Fornax does not span a large enough range of $[\mathrm{Fe} / \mathrm{H}]$ to draw definitive conclusions on the metallicity dependence of $[\mathrm{C} / \mathrm{Fe}]_{\text {corr }}$.

We considered the possibility that $[\mathrm{C} / \mathrm{Fe}]_{\text {corr }}$ appears to decline with $[\mathrm{Fe} / \mathrm{H}]$ because our spectra lack the $\mathrm{S} / \mathrm{N}$ or resolution to measure carbon abundances at low metallicities. We calculated upper limits for all of the stars where we could not measure carbon abundances. If the $[\mathrm{C} / \mathrm{Fe}]_{\text {corr }}$ trend appeared because of the loss of measurability at low $[\mathrm{Fe} / \mathrm{H}]$, then the upper limits would trace the lower envelope of $[\mathrm{C} / \mathrm{Fe}]_{\text {corr }}$ measurements. Instead, the upper limits are distributed fairly evenly in $[\mathrm{C} / \mathrm{Fe}]_{\text {corr }}$. We conclude that the decline is real.

The decline of $[\mathrm{C} / \mathrm{Fe}]_{\text {corr }}$ with $[\mathrm{Fe} / \mathrm{H}]$ can be explained by the varying contribution of different nucleosynthetic sources over time. Figure 10 shows theoretical nucleosynthetic yields superimposed on the same data as in Figure 9. We adopted the AGB yields of Karakas (2010), the SN II yields of Nomoto et al. (2006), and the SN Ia yields of Iwamoto et al. (1999, model W7).

$\mathrm{SNe}$ II are the first sources to enrich a galaxy with $\mathrm{C}$ and $\mathrm{Fe}$. Figure 10 shows the SN II yields averaged over a Salpeter (1955) initial mass function (IMF). In practice, the most massive SNe explode first. According to Nomoto et al. (2006), a $40 M_{\odot} \mathrm{SN}$ has a $\mathrm{C} / \mathrm{Fe}$ yield 5 times that of a $13 M_{\odot} \mathrm{SN}$ $([\mathrm{C} / \mathrm{Fe}]=+0.36$ versus -0.36$)$. As a result, the very earliest stars in the galaxy may have been able to incorporate the ejecta of only the most massive, shortest lived $\mathrm{SNe}$, which have the highest $[\mathrm{C} / \mathrm{Fe}]$ of the SNe II. The two stars in our sample with $[\mathrm{Fe} / \mathrm{H}]<-3$, both in Draco, have $[\mathrm{C} / \mathrm{Fe}]_{\text {corr }}=-0.39 \pm 0.37$ and $+0.76 \pm 0.13$. The difference between these values could indicate separate enrichment from SNe II of different masses, or it could indicate different levels of AGB pollution.

The IMF-averaged SN II yields are lower than the observed $[\mathrm{C} / \mathrm{Fe}]$ in the majority of $\mathrm{dSph}$ stars at $[\mathrm{Fe} / \mathrm{H}] \lesssim-1.7$. These stars require a more carbon-rich nucleosynthetic source, such as AGB stars. AGB stars were likely polluting the interstellar medium even at the very lowest metallicities. Karakas (2010) did not calculate AGB yields at $[\mathrm{Fe} / \mathrm{H}]<-2.3$, but AGB stars surely existed at lower metallicities. If the high $[\mathrm{C} / \mathrm{Fe}]$ yields persist at $[\mathrm{Fe} / \mathrm{H}]<-2.3$, then AGB ejecta could easily explain the high $[\mathrm{C} / \mathrm{Fe}]$ values observed at low $[\mathrm{Fe} / \mathrm{H}]$ in the dSphs. Their ejecta would mix with SN II ejecta to produce the $[\mathrm{C} / \mathrm{Fe}]$ ratio observed in low-metallicity stars. Because AGB stars have much larger $[\mathrm{C} / \mathrm{Fe}]$ ratios than $\mathrm{SNe}$ II, it would not take very many AGB stars compared to SNe II to explain the carbon measurements in low-metallicity dSph stars. 


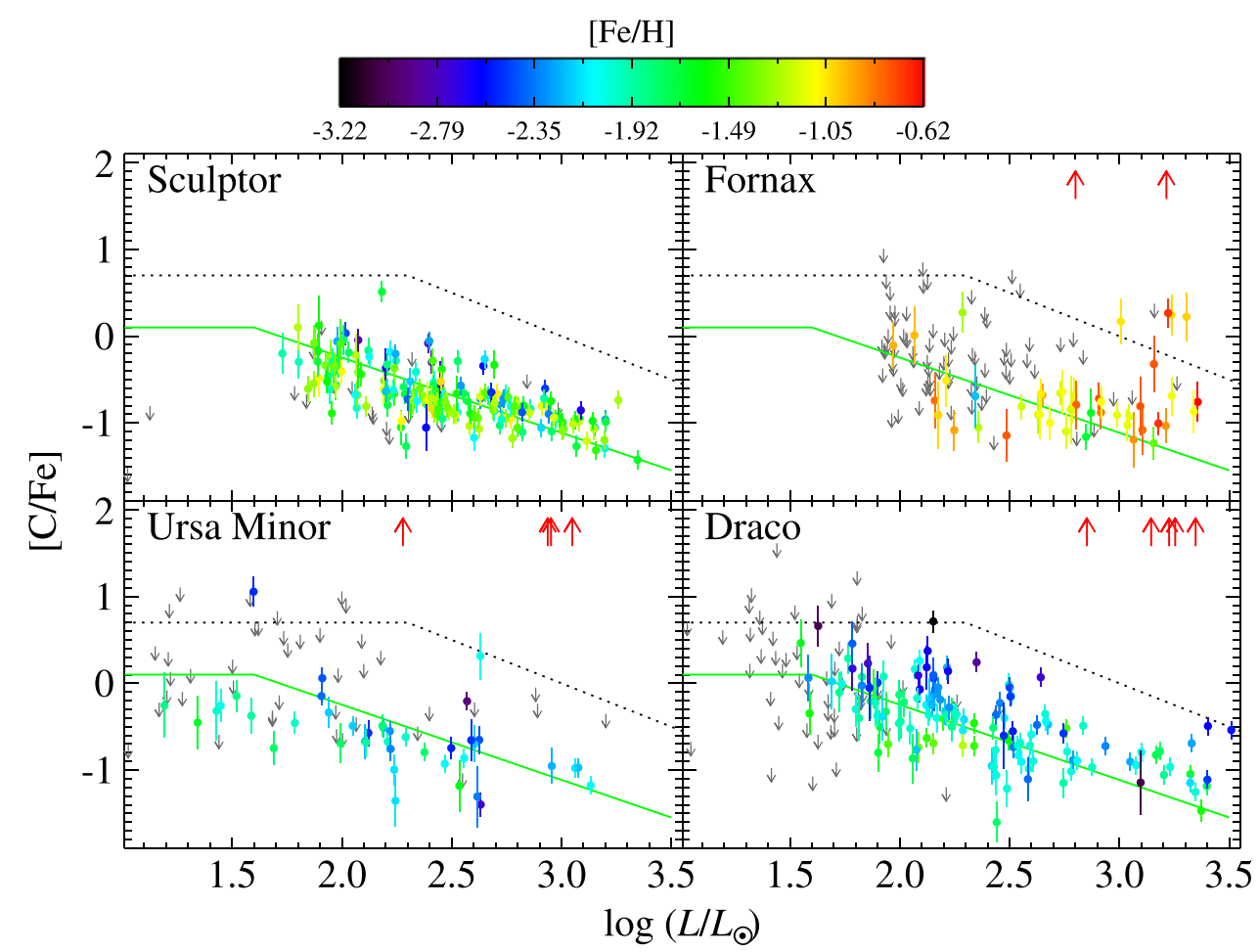

Figure 8. Carbon enhancement as a function of stellar luminosity in dSph stars. The green line (Equation (2)), copied from the GC data in Figure 6, shows the destruction of carbon as photospheric material is processed through the CNO cycle on the upper RGB. The color coding corresponds to iron abundance. The dotted line is Aoki et al.'s (2007) dividing line for carbon enhancement. Upward-pointing red arrows indicate very carbon rich stars for which we did not attempt to measure $[\mathrm{C} / \mathrm{Fe}]$. Downward-pointing gray arrows are $2 \sigma$ upper limits.

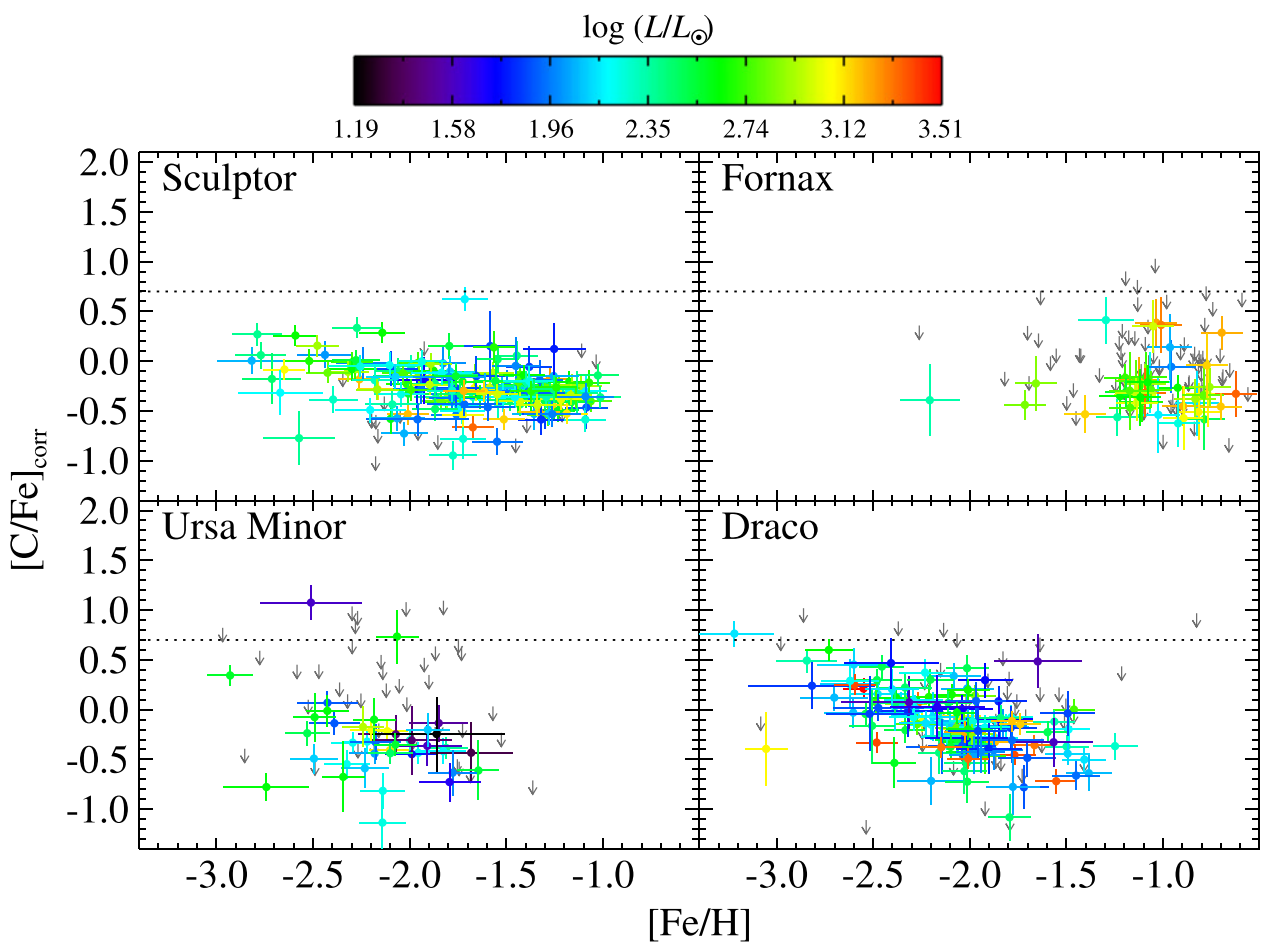

Figure 9. Carbon enhancement corrected for luminosity according to Placco et al. (2014) as a function of stellar metallicity. The color coding corresponds to stellar luminosity. The dotted line is one of Placco et al.'s definitions for carbon enhancement. Downward-pointing gray arrows are $2 \sigma$ upper limits. The C stars are not plotted because we did not measure $[\mathrm{Fe} / \mathrm{H}]$ for those stars.

The AGB yields are highly metallicity-dependent. As the galaxy's metallicity increases, the AGB stars eject more and more $\mathrm{Fe}$ but about the same amount of C. From $\log \left(Z / Z_{\odot}\right)=-2.3$ to -0.7 , the $\mathrm{C}$ output decreases by a factor of 2 while the Fe output increases by a factor of 37 . Hence, the metallicity dependence of AGB yields could explain some of the decline in $[\mathrm{C} / \mathrm{Fe}]$ with increasing $[\mathrm{Fe} / \mathrm{H}]$. 


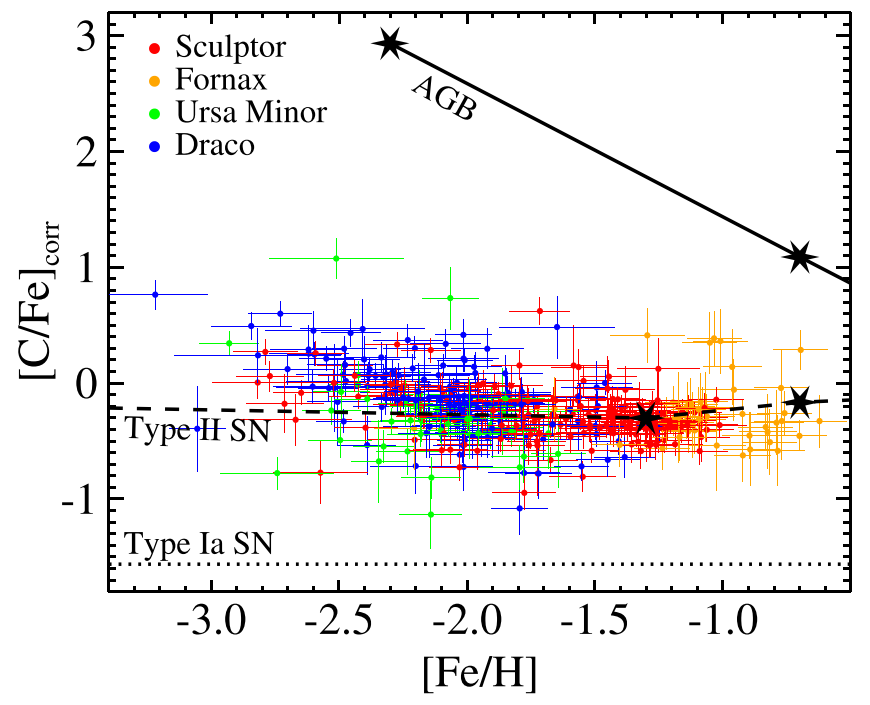

Figure 10. Carbon enhancement corrected for luminosity according to Placco et al. (2014) for all four dSphs. The theoretically predicted, IMF-averaged yields for nucleosynthetic sources are also shown: AGB stars (solid line, Karakas 2010), SNe II (dashed line, Nomoto et al. 2006), and SNe Ia (dotted line, Iwamoto et al. 1999). The AGB and SN II yields depend on metallicity. AGB stars could be active at $[\mathrm{Fe} / \mathrm{H}]<-2.3$, but Karakas's models are not available at lower $[\mathrm{Fe} / \mathrm{H}]$. Six-pointed symbols indicate the metallicities at which the yields were computed. A decline in $[\mathrm{C} / \mathrm{Fe}]_{\text {corr }}$ can be explained by the metallicity dependence of AGB yields combined with a growing contribution of SNe Ia.

$\mathrm{SNe}$ Ia are the last nucleosynthetic sources to turn on in $\mathrm{dSphs}$ because they are the end points of some long-lived, lowmass stars. The $\mathrm{SNe}$ Ia $[\mathrm{C} / \mathrm{Fe}]$ yield is -1.6 , significantly below the yield of SNe II and AGB stars. As the galaxy evolves, the ratio of $\mathrm{SNe}$ Ia to $\mathrm{SNe}$ II increases. Because the abundance ratios of the two SN types are drastically different, the trend of abundance ratios with $[\mathrm{Fe} / \mathrm{H}]$ reflects the changing contribution of the two SN types to the galaxy's chemical evolution (e.g., Gilmore \& Wyse 1991). The elements typically used to diagnose this ratio are $\alpha$ elements $(\mathrm{O}, \mathrm{Mg}$, and $\mathrm{Si}$ among others) and Fe. As Figure 10 demonstrates, $\mathrm{C} / \mathrm{Fe}$ is also sensitive to the Type II-to-Type Ia $\mathrm{SN}$ ratio. Although the heavy production of $\mathrm{C}$ in $\mathrm{AGB}$ stars complicates the interpretation of the $\mathrm{C} / \mathrm{Fe}$ ratio, some of the decline in $[\mathrm{C} / \mathrm{Fe}]$ with increasing $[\mathrm{Fe} / \mathrm{H}]$ in $\mathrm{dSphs}$ must be due to the increasing frequency of SNe Ia with time.

The four dSphs show different $[\mathrm{C} / \mathrm{Fe}]$ trends. The $[\mathrm{C} / \mathrm{Fe}]$ ratio declines most steeply in Ursa Minor and Draco. Under the presumption that an increasing prevalence of SNe Ia drives the decline in $[\mathrm{C} / \mathrm{Fe}]$, then these two dSphs would have had a very inefficient SFH. The galaxy would have taken about $100 \mathrm{Myr}$ - the approximate delay time for a $\mathrm{SNe} \mathrm{Ia}$ - to reach $[\mathrm{Fe} / \mathrm{H}] \approx-2.5$, the metallicity at which $[\mathrm{C} / \mathrm{Fe}]$ begins to decline. Unfortunately, Ursa Minor, Draco, and Sculptor are too old to permit fine time resolution in measurements of their SFHs (e.g., Orban et al. 2008), so our chemical interpretation cannot be cross-checked against photometrically measured SFHs. Finally, Fornax has a complex SFH (e.g., de Boer et al. 2012). Its pattern of $[\mathrm{C} / \mathrm{Fe}]$ is unlike the other three dSphs. Some of the most metal-rich stars have high values of $[\mathrm{C} / \mathrm{Fe}]_{\text {corr }}$. These measurements also have some the largest uncertainties, largely because the spectrum at $4300 \AA$ is blanketed with atomic and molecular absorption lines. If these measurements are trustworthy, then we speculate that AGB stars are responsible for the high carbon abundances, possibly through mass transfer.

We conclude that the primary causes of the decline of $[\mathrm{C} / \mathrm{Fe}]_{\text {corr }}$ with $[\mathrm{Fe} / \mathrm{H}]$ are $(1)$ the metallicity dependence of AGB yields and (2) the increasing ratio of Type Ia to Type II $\mathrm{SNe}$ with time. A quantitative interpretation of Figures 9 and 10 would require a chemical evolution model. Kirby et al. (2011) developed such a model to explain the decline of $[\alpha / \mathrm{Fe}]$ with $[\mathrm{Fe} / \mathrm{H}]$ in $\mathrm{dSphs}$, including the four $\mathrm{dSphs}$ discussed here. One prospect for future work is to incorporate carbon into the model, paying close attention to the yields of AGB stars.

Metallicity does not completely explain the observed scatter in $[\mathrm{C} / \mathrm{Fe}]$. Gratton et al. (2000) observed a large scatter of $[\mathrm{C} / \mathrm{Fe}]$ on the upper RGB in low-metallicity field stars in the MW halo. However, they did not have a large enough sample of unevolved stars at low metallicity to determine whether the scatter was imparted to the stars at birth or if it was caused by star-to-star variations in the efficiency of carbon astration on the upper RGB. Spite et al. (2005) found an approximately constant value of $[\mathrm{C} / \mathrm{Fe}]$ in unevolved field stars (ignoring $\mathrm{C}$ stars), even at low metallicities. This suggests that lowmetallicity, star-forming gas in the early MW halo had a uniform $[\mathrm{C} / \mathrm{Fe}]$ ratio. However, Spite et al. (2006) found that evolved stars on the upper RGB did not all experience the same level of mixing. In particular, the $[(\mathrm{C}+\mathrm{N}) / \mathrm{Fe}]$ ratio was larger for evolved stars at $[\mathrm{Fe} / \mathrm{H}]<-2.5$ than for higher-metallicity stars. In CNO-processed material that did not undergo much of the high-temperature $\mathrm{ON}$ cycle, the $[(\mathrm{C}+\mathrm{N}) / \mathrm{Fe}]$ ratio should be constant. Hence, Spite et al. (2006) uncovered a metallicity dependence in the efficiency of mixing.

In principle, Placco et al.'s (2014) metallicity-dependent carbon corrections should account for the variation in mixing efficiencies found by Spite et al. (2006). However, we still observe a dispersion in $[\mathrm{C} / \mathrm{Fe}]_{\text {corr }}$ beyond the measurement uncertainty at fixed $[\mathrm{Fe} / \mathrm{H}]$. We fit a quadratic relation between $[\mathrm{C} / \mathrm{Fe}]_{\text {corr }}$ and $[\mathrm{Fe} / \mathrm{H}]$ to stars with $[\mathrm{C} / \mathrm{Fe}]_{\text {corr }}<+0.7$ (to exclude stars that may have acquired carbon through binary mass transfer). In a manner similar to calculating the intrinsic dispersion of $[\mathrm{C} / \mathrm{Fe}]$ in GCs (Section 4), we calculated the residuals, $\Delta$, about the quadratic fit and divided them by the measurement uncertainties: $\Delta=\left([\mathrm{C} / \mathrm{Fe}]_{\text {corr }}-[\mathrm{C} / \mathrm{Fe}]_{\text {corr }}(\right.$ fit $\left.)\right) /$ $\delta[\mathrm{C} / \mathrm{Fe}]$. If measurement uncertainties explain the scatter, then the standard deviation of $\Delta$ should be 1 . Instead, we found standard deviations of 1.5 for Sculptor, 1.2 for Fornax, 1.5 for Ursa Minor, and 1.6 for Draco. Therefore, all of the dSphs show a small residual beyond the measurement uncertainties that cannot be explained by the decline of $[\mathrm{C} / \mathrm{Fe}]$ with increasing stellar luminosity (Figure 8) or with increasing metallicity (Figure 9).

One possible source of the residual scatter is inhomogeneous mixing (e.g., Revaz \& Jablonka 2012). If different contemporaneous star formation regions formed out of gas that was not mixed across regions, then stars at the same metallicity and luminosity could have different abundance ratios. Also, the calculation of $\Delta$ depends on accurate estimates of measurement uncertainty. If we have underestimated $\delta[\mathrm{C} / \mathrm{Fe}]$, then we have also overestimated $\Delta$. Another possibility is that Placco et al.'s (2014) corrections are imperfect. Errors on the order of $\sim 0.15$ dex in the corrections could cause the scatter in $[\mathrm{C} / \mathrm{Fe}]_{\text {corr }}$ that we observe. 
Table 5

Carbon Stars

\begin{tabular}{lllc}
\hline \hline DSph & Name & Reference & Reference ID \\
\hline Fornax & 50465 & This paper & $\ldots$ \\
Fornax & 98788 & This paper & $\ldots$ \\
Ursa Minor & Bel60031 & Aaronson et al. (1983) & 34227 \\
$\ldots$ & $\ldots$ & Armandroff et al. (1995) & VA335 \\
Ursa Minor & Bel60230 & This paper & $\ldots$ \\
Ursa Minor & Bel80022 & Shetrone et al. (2001) & UM 1545 \\
Ursa Minor & Bel10023 & Canterna \& Schommer (1978) & COS215 \\
$\ldots$ & $\ldots$ & Zinn (1981) & K \\
$\ldots$ & $\ldots$ & Aaronson et al. (1983) & K \\
$\ldots$ & $\ldots$ & Armandroff et al. (1995) & K \\
$\ldots$ & $\ldots$ & Shetrone et al. (2001) & K \\
$\ldots$ & $\ldots$ & Winnick (2003) & COS215 \\
Draco & 670092 & Armandroff et al. (1995) & 461 \\
Draco & 569394 & Aaronson et al. (1982) & 3203 \\
$\ldots$ & $\ldots$ & Armandroff et al. (1995) & C1 \\
Draco & 607050 & Aaronson et al. (1982) & J \\
$\ldots$ & $\ldots$ & Armandroff et al. (1995) & C2 \\
Draco & 571725 & Aaronson et al. (1982) & 3237 \\
$\ldots$ & $\ldots$ & Armandroff et al. (1995) & C3 \\
Draco & 682522 & Azzopardi et al. (1986) & 578 \\
$\ldots$ & $\ldots$ & Armandroff et al. (1995) & C4 \\
\hline & & $\ldots$ & $\ldots$
\end{tabular}

\subsection{Carbon Stars}

As demonstrated in M15 (Section 4), stars need not be born with their present $[\mathrm{C} / \mathrm{Fe}]$ ratio. They can acquire large amounts of carbon through mass transfer from an AGB star. These stars can exhibit $[\mathrm{C} / \mathrm{Fe}] \gg+1$. We identified such stars in our dSph sample. We found $2 \mathrm{C}$ stars in Fornax, 4 in Ursa Minor, 5 in Draco, and none in Sculptor. They are represented as red, five-pointed symbols in Figure 7. Most of these stars were discovered in previous surveys. ${ }^{19}$ Table 5 lists all of the $\mathrm{C}$ stars in our sample along with the the reference(s) and identification name(s) for their previous discoveries, where applicable. They all have extremely strong $\mathrm{CN}$ bands, as shown in Figure 2. Most of the $C$ stars also have very strong $\mathrm{C}_{2}$ bands visible in the blue spectra. However, two of the three Ursa Minor $\mathrm{C}$ stars have weak $\mathrm{C}_{2}$, and $\mathrm{C}_{2}$ is not visible at all in star Bel80022. It is likely too metal-poor and perhaps too warm for $\mathrm{C}_{2}$ to appear in our low-resolution spectrum.

The resolution of our DEIMOS spectra does not permit a detailed chemical abundance analysis of these carbon stars. The $\mathrm{CN}, \mathrm{C}_{2}$, and $\mathrm{CH}$ molecular bands blanket the spectra too heavily. For this reason, their carbon abundances are represented as lower limits in Figure 8. They are not represented at all in Figure 9 because we could not measure $[\mathrm{Fe} / \mathrm{H}]$. Higher resolution spectra would allow a detailed chemical analysis (e.g., Karinkuzhi \& Goswami 2014). Almost all of the carbon stars we found are in fact bright enough to be observed with a high-resolution spectrograph on a large telescope, like Keck/HIRES. We reserve this task for future work.

Figure 7 shows that five of the $11 \mathrm{C}$ stars are redward of the RGB. The tendency for carbon stars (or more specifically, barium stars, which almost always have excess carbon) to appear red in optical colors is called the Bond-Neff effect

\footnotetext{
${ }^{19}$ Shetrone et al. (2001) identified star 622253 (called 68 in their paper) in Draco as a $\mathrm{C}$ star. We do not find it to be carbon-rich. We measured its carbon enhancement to be $[\mathrm{C} / \mathrm{Fe}]=-0.52 \pm 0.11$.
}

(Bond \& Neff 1969). The source of the effect is a depression of blue flux due to molecular blanketing of the spectrum. In fact, all five of the $\mathrm{C}$ stars redder than the RGB show strong $\mathrm{C}_{2}, \mathrm{CH}$, and $\mathrm{CN}$

Our spectroscopic target selection was focused on selecting stars from the RGB. Hence, we may have missed many $\mathrm{C}$ stars. Although we discuss the $\mathrm{C}$ stars in our sample, the sample is definitely not complete. Other surveys that specifically searched for C stars (e.g., Demers \& Battinelli 2002) are better able to quantify their frequency. Our sample selection for Sculptor was especially biased against finding $\mathrm{C}$ stars. Skúladóttir et al. (2015) found the first CEMP star in Sculptor, but it lies outside of the area we surveyed.

The brightest C star in Ursa Minor, Bel10023, is actually blueward of the RGB. The molecular absorption apparently is not strong enough to redden the optical colors. Indeed, the $\mathrm{C}_{2}$ bands in this star are visible but very weak. The star is probably an AGB star, just like 47866 in M15. It possibly manufactured its carbon by itself rather than acquiring it through binary mass transfer, though the star is probably too low-mass to dredge up carbon. Unfortunately, our spectra do not provide any clues as to whether the $\mathrm{C}$ stars generated their carbon themselves or acquired it externally.

\section{COMPARISON TO THE MILKY WAY STELLAR HALO}

The MW stellar halo most likely grows by the minor merging of satellite galaxies. As a result, the chemical abundance distribution of the halo is the superposition of the abundance distributions of all of its constituents. The abundances of these individual satellites depend on their stellar masses and SFHs. Therefore, the mass distribution and SFH of the halo's dissolved satellites can be inferred from the chemical abundances of the halo (see Lee et al. 2014).

One straightforward way to compare the carbon abundance distributions of the surviving $\mathrm{dSphs}$ to the $\mathrm{MW}$ halo is to measure the frequency of CEMP stars. The frequency depends on metallicity. Specifically, it decreases toward higher metallicity (Beers et al. 1992). In Section 5, we showed that $[\mathrm{C} / \mathrm{Fe}]$ does indeed decrease with increasing $[\mathrm{Fe} / \mathrm{H}]$. After accounting for this decrease with metallicity, Starkenburg et al. (2013) presented evidence that the CEMP fraction in Sculptor is lower than in the MW halo with $290 \%$ confidence. This result would suggest that Sculptor had a different chemical evolution or perhaps a different frequency of mass-transfer binaries than halo progenitors.

Very few of the stars in our sample would qualify as CEMP under the various definitions for "CEMP." One proposed definition is a simple cut in carbon abundances, such as $[\mathrm{C} / \mathrm{Fe}]>+1.0$ (Beers \& Christlieb 2005). However, this definition disregards the destruction of carbon on the upper RGB, which is the location for all of the stars in our sample. A more nuanced definition accounts for the luminosity of the star (Aoki et al. 2007; Yong et al. 2013; Placco et al. 2014). We consider the CEMP fraction with two different definitions. First, we consider Aoki et al.'s (2007) definition:

$$
\begin{array}{ll}
{[\mathrm{C} / \mathrm{Fe}] \geqslant+0.7} & \text { if } \log \left(L / L_{\odot}\right) \leqslant 2.3 \\
{[\mathrm{C} / \mathrm{Fe}] \geqslant+3.0-\log \left(L / L_{\odot}\right)} & \text { if } \log \left(L / L_{\odot}\right)>2.3
\end{array}
$$

The dotted line in Figure 8 shows the dividing line of Equation (3). 
We identified only 6 CEMP stars out of 398 stars in our dSph sample using Aoki et al.'s (2007) definition of CEMP. Of these, 1 has $[\mathrm{Fe} / \mathrm{H}] \leqslant-3.0,2$ have $[\mathrm{Fe} / \mathrm{H}] \leqslant-2.5$, and 5 have $[\mathrm{Fe} / \mathrm{H}] \leqslant-1.0$. In these same metallicity ranges, the CEMP fractions are $(50 \pm 50) \%,(7.1 \pm 5.1) \%$, and $(1.3 \pm 0.6) \%$. However, our sample is heavily biased against extremely carbon-rich stars. First, we selected against stars redward of the RGB, where $\mathrm{C}$ stars are generally found. Second, our technique of measuring $[\mathrm{Fe} / \mathrm{H}]$ in the range $6300-9100 \AA$ fails for extremely carbon-rich stars, in which $\mathrm{CN}$ blankets much of that spectral region. We found $11 \mathrm{C}$ stars, but we did not measure their metallicities. As a result, they are not included in the above estimate of CEMP frequency. Therefore, the frequencies of carbon stars we found in dSphs should be regarded strictly as lower limits.

We also counted CEMP stars using $[\mathrm{C} / \mathrm{Fe}]_{\text {corr }}$, which is corrected for luminosity according to Placco et al. (2014). One of Placco et al.'s proposed definitions for CEMP is $[\mathrm{C} / \mathrm{Fe}]_{\text {corr }}>+0.7$ (dotted line in Figure 9). The cut does not need any dependence on luminosity because $[\mathrm{C} / \mathrm{Fe}]_{\text {corr }}$ already depends on luminosity. Under this definition, we found only 3 out of 398 stars. They have $[\mathrm{Fe} / \mathrm{H}]=-3.22 \pm 0.20$, $-2.51 \pm 0.26$, and $-2.07 \pm 0.11$.

The CEMP fraction of the halo is significantly higher than the lower limits we determined for dSphs except in the $[\mathrm{Fe} / \mathrm{H}] \leqslant-3.0$ range, where we observed only 2 stars. In the halo, the CEMP fraction is approximately $13 \%$ at $[\mathrm{Fe} / \mathrm{H}] \leqslant-2$ and $25 \%$ at $[\mathrm{Fe} / \mathrm{H}] \leqslant-3$ (Cohen et al. 2005; Marsteller et al. 2005; Lucatello et al. 2006; Frebel et al. 2006; Carollo et al. 2012; Yong et al. 2013; Lee et al. 2013; Placco et al. 2014). Many of the halo's CEMP stars have $[\mathrm{C} / \mathrm{Fe}] \gtrsim+2$. These stars would have been excluded from our sample.

Nonetheless, we can still compare the general trend of $[\mathrm{C} / \mathrm{Fe}]$ versus $[\mathrm{Fe} / \mathrm{H}]$ for stars with $[\mathrm{C} / \mathrm{Fe}]<+1$ between $\mathrm{dSphs}$ and the halo. Although our sample misses many of the stars with $[\mathrm{C} / \mathrm{Fe}] \gtrsim+1$, the remaining stars show a trend different from the MW halo, as shown in Figure 11. The top panel compares our measurements of $[\mathrm{C} / \mathrm{Fe}]_{\text {corr }}$ in $\mathrm{dSphs}$ compared to highresolution measurements in the MW halo (Gratton et al. 2000; Spite et al. 2005). The sample of Gratton et al. includes dwarfs and giants, and the sample of Spite et al. is only giants. We applied Placco et al.'s (2014) corrections to the $[\mathrm{C} / \mathrm{Fe}]$ measurements in both samples. The bottom panel compares our measurements to Sloan Extension for Galactic Understanding and Exploration (SEGUE) main sequence turn-off stars (Lee et al. 2013), measured by SEGUE (Yanny et al. 2009). We plot the turn-off stars because their carbon abundances have not yet been affected by stellar astration. We adjusted all of the MW [C/Fe] measurements to match the solar abundance ratios we adopted (see Section 3.4.3). Ignoring the CEMP stars, the $[\mathrm{C} / \mathrm{Fe}]$ pattern of the general halo population is flat at $[\mathrm{C} / \mathrm{Fe}] \sim+0.1$ until $[\mathrm{Fe} / \mathrm{H}]=-0.7$, whereupon it drops to $[\mathrm{C} / \mathrm{Fe}] \sim-0.2$ at $[\mathrm{Fe} / \mathrm{H}]=0$. In contrast, the dSph stars have already dropped to $[\mathrm{C} / \mathrm{Fe}] \sim-0.4$ by a metallicity of $[\mathrm{Fe} / \mathrm{H}]=-2$. Note that the floor in the SEGUE data at $[\mathrm{C} / \mathrm{Fe}] \sim-0.3$ might be artificial. The lowest value of $[\mathrm{C} / \mathrm{Fe}]$ in Lee et al.'s grid of synthetic spectra is -0.5 , and the minimum upper limit values (not plotted in Figure 11) are mostly about 0.2 dex away from the grid limit. Regardless, the SEGUE sample is qualitatively consistent with the samples of Gratton et al. (2000) and Spite et al. (2005).
Lee et al. (2015) interpreted the halo's CEMP fraction in the context of binary mass transfer. They found that carbon donation from AGB stars could explain the present $[\mathrm{C} / \mathrm{Fe}]$ distribution for giant stars. However, that mechanism is primarily responsible for large carbon enhancements $([\mathrm{C} / \mathrm{Fe}]>+1)$. In Section 5 and Figure 10, we interpreted the trend among non-CEMP stars in the context of galactic chemical evolution. The different $[\mathrm{C} / \mathrm{Fe}]$ slopes between the $\mathrm{dSph}$ and halo samples is reminiscent of the different slopes in $[\alpha / \mathrm{Fe}]$ (e.g., Shetrone et al. 1998a; Venn et al. 2004). The $[\alpha / \mathrm{Fe}]$ distribution of the halo shows a downturn (a "knee") at $[\mathrm{Fe} / \mathrm{H}]=-0.7$, a feature that is also visible in $[\mathrm{C} / \mathrm{Fe}]$ (Figure 11). The knee corresponds to the metallicity at which $\mathrm{SNe}$ Ia began to pollute the MW. Because SNe Ia have low yields of both $[\alpha / \mathrm{Fe}]$ and $[\mathrm{C} / \mathrm{Fe}]$, their prevalence beginning at $[\mathrm{Fe} / \mathrm{H}]=-0.7$ can explain the abundance pattern for both abundance ratios in the halo. However, the knee happens at lower metallicities for dSphs. The decline of $[\mathrm{C} / \mathrm{Fe}]$ with increasing $[\mathrm{Fe} / \mathrm{H}]$ begins at $[\mathrm{Fe} / \mathrm{H}] \lesssim-2.5$ for Ursa Minor and Draco. The lower metallicity of their $[\mathrm{C} / \mathrm{Fe}]$ knees indicates that $\mathrm{SNe}$ Ia were active at much lower metallicities in dSphs than in the halo. The same trend is seen in dSphs' $[\alpha / \mathrm{Fe}]$ ratios (e.g., Venn et al. 2004; Kirby et al. 2011). The halo reached a much higher metallicity than the dSphs before $\mathrm{SNe}$ Ia turned on. Furthermore, both $[\mathrm{C} / \mathrm{Fe}]$ and $[\alpha / \mathrm{Fe}]$ in $\mathrm{dSphs}$ decline to values well below the solar ratio, indicating a heavy dose of $\mathrm{SNe}$ Ia relative to the halo, which has larger values of $[\mathrm{C} / \mathrm{Fe}]$ and $[\alpha / \mathrm{Fe}]$. Our $[\mathrm{C} / \mathrm{Fe}]$ measurements are further proof that the chemical evolution of surviving dSphs was much slower than the progenitors of the MW halo.

\section{SUMMARY}

Carbon is a complex element. It has diverse nucleosynthetic sources. AGB stars produce copious amounts of carbon, but their $\mathrm{C} / \mathrm{Fe}$ ratios depend sensitively on metallicity and stellar mass. SNe II produce some carbon, whereas $\mathrm{SNe}$ Ia produce very little carbon but a great deal of iron. The timescale for all three sources is different, which makes the $\mathrm{C} / \mathrm{Fe}$ ratio change as the galaxy evolves in a way that depends on the details of the SFH. Mass transfer from past AGB stars further complicates the interpretation of the carbon abundances of present-day stars. In particular, some stars in mass-transfer binaries can have orders of magnitude more carbon than single stars of the same metallicity. In addition to multiple sources, carbon also has a sink. Red giants astrate carbon on the upper RGB. The rate of astration depends on the metallicity and luminosity of the star.

In order to explore the evolution of carbon, we observed 745 red giants with the Keck/DEIMOS spectrograph. We generated a grid of model spectra based on a line list that we checked against spectra of the Sun and Arcturus. We measured carbon abundances by fitting the model spectra to the $\mathrm{G}$ band at $4300 \AA$. We were able to measure $[\mathrm{C} / \mathrm{Fe}]$ for 552 stars. We validated our measurements by comparing a subset of our observations to published measurements of carbon abundances for the same stars. We found offsets between our measurements of $[\mathrm{C} / \mathrm{Fe}]$ and the literature measurements. However, the offset depended on the source of the measurements. Our measurements agreed most closely with those based on atomic and molecular line lists most similar to our own.

GCs provide controlled environments to study the evolution of carbon because they generally have single ages and 


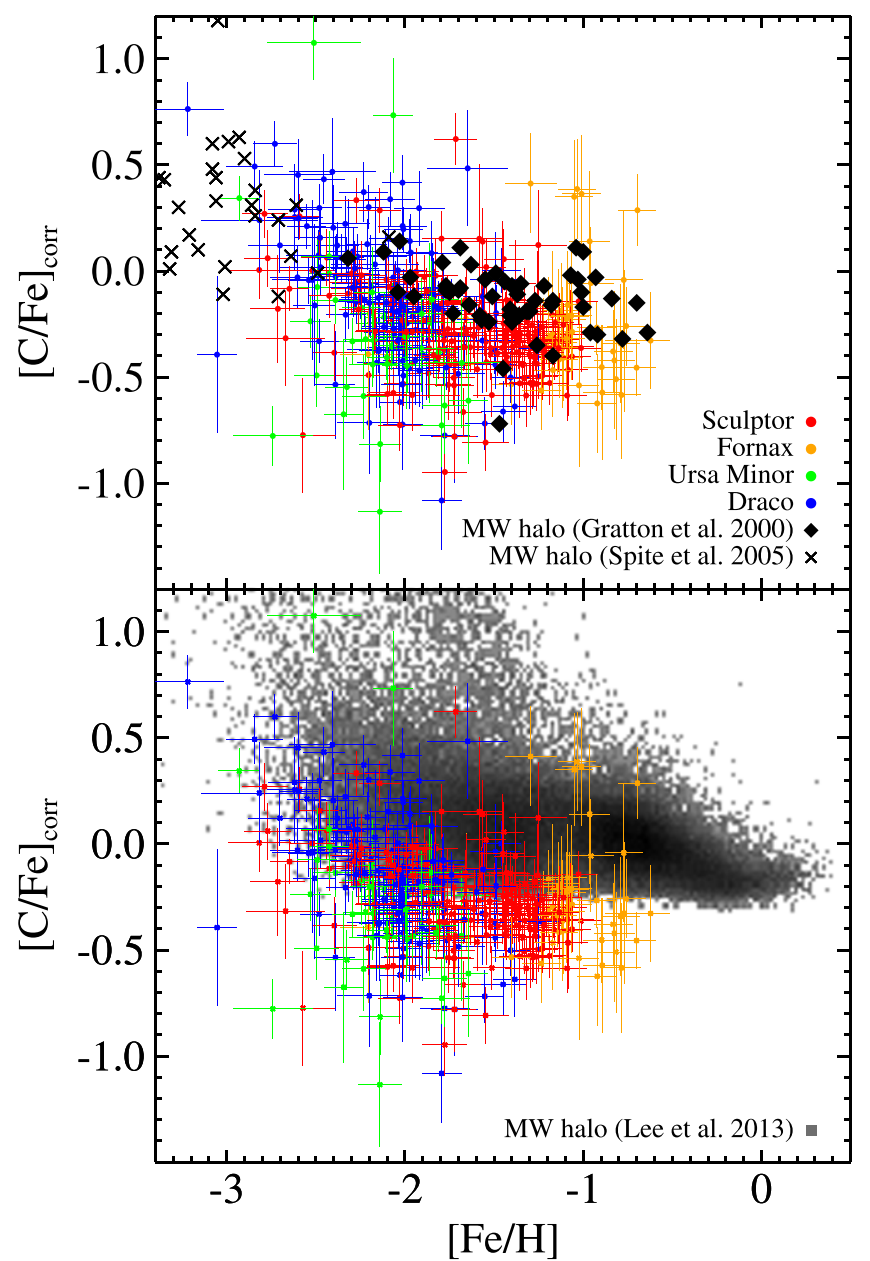

Figure 11. The trend of $[\mathrm{C} / \mathrm{Fe}]_{\text {corr }}$ vs. $[\mathrm{Fe} / \mathrm{H}]$ in dSphs compared to stars in the MW (top panel: Gratton et al. 2000 and Spite et al. 2005; bottom panel: Lee et al. 2013). In the bottom panel, the grayscale for MW stars is logarithmic in number density. The bottom panel shows only main sequence turn-off stars, which were selected in the same manner as in Lee et al.: $5600 \mathrm{~K} \leqslant T_{\text {eff }} \leqslant 6700 \mathrm{~K}$. These stars have not altered their surface compositions, so their $[\mathrm{C} / \mathrm{Fe}]$ measurements do not need to be corrected.

metallicities. We measured $[\mathrm{C} / \mathrm{Fe}]$ in 154 red giants in the $\mathrm{GCs}$ NGC 2419, M68, and M15. We observed the well-known decline of $[\mathrm{C} / \mathrm{Fe}]$ on the upper RGB starting at the luminosity function bump. We found that carbon does not participate in the unusual abundance patterns in NGC 2419 (Cohen \& Kirby 2012; Mucciarelli et al. 2012). We also reported the discovery of a carbon-enhanced AGB star (47866) in M15.

We also explored the evolution of carbon in MW dSphs by examining 398 carbon abundances and 182 upper limits for stars confirmed to be members of Sculptor, Fornax, Ursa Minor, and Draco. As expected, the $[\mathrm{C} / \mathrm{Fe}]$ distribution is more complex in dSphs than in GCs. Unlike GCs, dSphs show a dispersion in $[\mathrm{C} / \mathrm{Fe}]$ at fixed stellar luminosity. The dispersion depends on $[\mathrm{Fe} / \mathrm{H}]$. Specifically, stars with lower $[\mathrm{Fe} / \mathrm{H}]$ have higher $[\mathrm{C} / \mathrm{Fe}]$ than stars at the same luminosity with higher $[\mathrm{Fe} / \mathrm{H}]$. We interpreted the trend with metallicity as an evolution in the influence of the three sources of carbon. First, SNe II seeded the dSph with the first C and Fe. Very soon after, the first AGB stars produced a great deal of $\mathrm{C}$ relative to Fe. Finally, SNe Ia produced very little $\mathrm{C}$ but a large amount of Fe after $\sim 100 \mathrm{Myr}$. The result is a decline in
$[\mathrm{C} / \mathrm{Fe}]$ with increasing $[\mathrm{Fe} / \mathrm{H}]$ that roughly tracks the decline in $[\alpha / \mathrm{Fe}]$, also due to SNe Ia (e.g., Kirby et al. 2011).

Our color selection biased the sample against very carbonrich stars. Nonetheless, we found $11 \mathrm{C}$ stars (eight previously known) in three dSphs. These stars are either AGB stars that are producing carbon now or the results of binary mass transfer from past AGB stars. Because of our selection bias, we calculated only lower limits to the CEMP fraction in dSphs. These lower limits are mostly below the CEMP fraction for the MW halo. Therefore, we have found no tension for the theory of hierarchical assembly, wherein the halo builds mass through minor merging of satellites like dSphs (e.g., Bullock \& Johnston 2005). However, this question would be addressed better by a spectroscopic survey of dSphs with a color cut more inclusive of stars redder than the RGB.

Our sample is still useful for comparing the trend of $[\mathrm{C} / \mathrm{Fe}]$ versus $[\mathrm{Fe} / \mathrm{H}]$ for carbon-normal stars. We found that the knee in the $[\mathrm{C} / \mathrm{Fe}]$ distribution occurs at a lower metallicity in dSphs than in the MW halo (Lee et al. 2013). This difference in metallicity is reminiscent of the different $[\alpha / \mathrm{Fe}]$ knees between dSphs and the MW halo (Venn et al. 2004). The similarity in behavior between $[\mathrm{C} / \mathrm{Fe}]$ and $[\alpha / \mathrm{Fe}]$ supports our hypothesis that $\mathrm{SNe}$ Ia are responsible for the decline in $[\mathrm{C} / \mathrm{Fe}]$. The $[\alpha / \mathrm{Fe}]$ and $[\mathrm{C} / \mathrm{Fe}]$ distributions together indicate that the progenitors of the MW halo had a more efficient SFH, which reached a higher metallicity before SNe Ia began to produce Fe in earnest.

Our sample of 398 measurements doubles the number of carbon abundances known in dSphs. Each dSph has enough carbon measurements (181 in Sculptor, 45 in Fornax, 41 in Ursa Minor, and 131 in Draco) for analysis with a numerical chemical evolution model (e.g., Revaz et al. 2009; Kirby et al. 2011). Although we have presented a qualitative interpretation for the observed carbon abundance trends, a numerical model will give a quantitative timescale for star formation. This chemical timescale can be compared to photometric measurements of SFH. Furthermore, numerical models can possibly constrain the IMF (Lee et al. 2015) or binary fraction (Lucatello et al. 2005) in dSphs. We invite interested modelers to make quantitative models of the carbon abundances in Table 4 to reveal the history of star formation in these four dSphs.

We thank Vinicius Placco for kindly computing astration corrections to carbon abundances. We also thank Chris Sneden for constructive comments on the structure of this paper and the anonymous referee for an insightful report. M.G., A.J.Z., and M.D. carried out their work through UCSC's Science Internship Program for high-school students. P.G. acknowledges support from NSF grants AST-1010039 and AST-1412648. We are grateful to the many people who have worked to make the Keck Telescope and its instruments a reality and to operate and maintain the Keck Observatory. The authors wish to extend special thanks to those of Hawaiian ancestry on whose sacred mountain we are privileged to be guests. Without their generous hospitality, none of the observations presented herein would have been possible.

Facility: Keck:II (DEIMOS)

\section{REFERENCES}

Aaronson, M., Hodge, P. W., \& Olszewski, E. W. 1983, ApJ, 267, 271

Aaronson, M., Liebert, J., \& Stocke, J. 1982, ApJ, 254, 507

Aaronson, M., \& Mould, J. 1980, ApJ, 240, 804 
Abia, C., de Laverny, P., \& Wahlin, R. 2008, A\&A, 481, 161

Alcaino, G., Liller, W., Alvarado, F., \& Wenderoth, E. 1990, AJ, 99, 1831

Anders, E., \& Grevesse, N. 1989, GeCoA, 53, 197

Aoki, W., Beers, T. C., Christlieb, N., et al. 2007, ApJ, 655, 492

Armandroff, T. E., Olszewski, E. W., \& Pryor, C. 1995, AJ, 110, 2131

Azzopardi, M., Lequeux, J., \& Westerlund, B. E. 1986, A\&A, 161, 232

Beers, T. C., \& Christlieb, N. 2005, ARA\&A, 43, 531

Beers, T. C., Preston, G. W., \& Shectman, S. A. 1992, AJ, 103, 1987

Behr, B. B., Cohen, J. G., McCarthy, J. K., \& Djorgovski, S. G. 1999, ApJL, 517, L135

Bell, R. A. 1985, PASP, 97, 219

Bellazzini, M., Ferraro, F. R., Origlia, L., et al. 2002, AJ, 124, 3222

Böhm-Vitense, E., Carpenter, K., Robinson, R., Ake, T., \& Brown, J. 2000, ApJ, 533, 969

Bond, H. E. 1975, ApJL, 202, L47

Bond, H. E., \& Neff, J. S. 1969, ApJ, 158, 1235

Briley, M. M., Cohen, J. G., \& Stetson, P. B. 2002, ApJL, 579, L17

Bullock, J. S., \& Johnston, K. V. 2005, ApJ, 635, 931

Canterna, R., \& Schommer, R. A. 1978, ApJL, 219, L119

Carbon, D. F., Romanishin, W., Langer, G. E., et al. 1982, ApJS, 49, 207

Carollo, D., Beers, T. C., Bovy, J., et al. 2012, ApJ, 744, 195

Charbonnel, C., \& Lagarde, N. 2010, A\&A, 522, A10

Charbonnel, C., \& Zahn, J.-P. 2007, A\&A, 467, L15

Cohen, J. G. 1999, AJ, 117, 2434

Cohen, J. G., \& Huang, W. 2009, ApJ, 701, 1053

Cohen, J. G., \& Huang, W. 2010, ApJ, 719, 931

Cohen, J. G., Huang, W., \& Kirby, E. N. 2011, ApJ, 740, 60

Cohen, J. G., \& Kirby, E. N. 2012, ApJ, 760, 86

Cohen, J. G., Persson, S. E., \& Frogel, J. A. 1978, ApJ, 222, 165

Cohen, J. G., Shectman, S., Thompson, I., et al. 2005, ApJL, 633, L109

Cohen, J. G., McWilliam, A., Shectman, S., et al. 2006, AJ, 132, 137

Cooper, M. C., Newman, J. A., Davis, M., Finkbeiner, D. P., \& Gerke, B. F. 2012, spec2d: DEEP2 DEIMOS Spectral Pipeline, Astrophysics Source Code Library

Côté, P., Hanes, D. A., McLaughlin, D. E., et al. 1997, ApJL, 476, L15

Cowley, A. P., \& Crampton, D. 1985, PASP, 97, 835

Dahn, C. C., Liebert, J., Kron, R. G., Spinrad, H., \& Hintzen, P. M. 1977, ApJ, 216, 757

de Boer, T. J. L., Tolstoy, E., Hill, V., et al. 2012, A\&A, 544, A73

Demarque, P., Woo, J.-H., Kim, Y.-C., \& Yi, S. K. 2004, ApJS, 155, 667

Demers, S., \& Battinelli, P. 2002, AJ, 123, 238

Dickens, R. J 1972, MNRAS, 159, 7P

Durrell, P. R., \& Harris, W. E. 1993, AJ, 105, 1420

Eggleton, P. P., Dearborn, D. S. P., \& Lattanzio, J. C. 2006, Sci, 314, 1580

Faber, S. M., Phillips, A. C., Kibrick, R. I., et al. 2003, in SPIE Conf. Ser. 4841, Instrument Design and Performance for Optical/Infrared Groundbased Telescopes, ed. M. Iye, \& A. F. M. Moorwood, 1657

Feast, M. W., Menzies, J. W., \& Whitelock, P. A. 2013, MNRAS, 428, L36

Font, A. S., Johnston, K. V., Bullock, J. S., \& Robertson, B. E. 2006, ApJ, 638,585

Frebel, A., Kirby, E. N., \& Simon, J. D. 2010a, Natur, 464, 72

Frebel, A., Simon, J. D., Geha, M., \& Willman, B. 2010b, ApJ, 708, 560

Frebel, A., Simon, J. D., \& Kirby, E. N. 2014, ApJ, 786, 74

Frebel, A., Christlieb, N., Norris, J. E., et al. 2006, ApJ, 652, 1585

Fulbright, J. P., Rich, R. M., \& Castro, S. 2004, ApJ, 612, 447

Gilmore, G., \& Wyse, R. F. G. 1991, ApJL, 367, L55

Girardi, L., Grebel, E. K., Odenkirchen, M., \& Chiosi, C. 2004, A\&A, 422, 205

Gratton, R., Sneden, C., \& Carretta, E. 2004, ARA\&A, 42, 385

Gratton, R. G., Sneden, C., Carretta, E., \& Bragaglia, A. 2000, A\&A, 354, 169

Harbeck, D., Smith, G. H., \& Grebel, E. K. 2003, AJ, 125, 197

Harding, G. A. 1962, Obs, 82, 205

Harris, W. E. 1996, AJ, 112, 1487

Harris, W. E., Bell, R. A., Vandenberg, D. A., et al. 1997, AJ, 114, 1030

Hilker, M., Kayser, A., Richtler, T., \& Willemsen, P. 2004, A\&A, 422, L9

Hinkle, K., Wallace, L., Valenti, J., \& Harmer, D. 2000, Visible and Near Infrared Atlas of the Arcturus Spectrum, 3727-9300

Iben, I., Jr. 1975, ApJ, 196, 525

Iwamoto, K., Brachwitz, F., Nomoto, K., et al. 1999, ApJS, 125, 439

Jorgensen, U. G., Larsson, M., Iwamae, A., \& Yu, B. 1996, A\&A, 315, 204

Karakas, A. I. 2010, MNRAS, 403, 1413

Karakas, A. I., García-Hernández, D. A., \& Lugaro, M. 2012, ApJ, 751, 8

Karinkuzhi, D., \& Goswami, A. 2014, MNRAS, 440, 1095

Keller, L. D., Pilachowski, C. A., \& Sneden, C. 2001, AJ, 122, 2554

Kinman, T. D., Carbon, D. F., Suntzeff, N., \& Kraft, R. P. 1981, in IAU Coll. 68, Astrophysical Parameters for Globular Clusters, ed. A. G. D. Philip, \& D. S. Hayes (Cambridge: Cambridge Univ. Press), 451
Kirby, E. N. 2011, PASP, 123, 531

Kirby, E. N., Cohen, J. G., Smith, G. H., et al. 2011, ApJ, 727, 79

Kirby, E. N., Guhathakurta, P., Bolte, M., Sneden, C., \& Geha, M. C. 2009, ApJ, 705, 328

Kirby, E. N., Guhathakurta, P., \& Sneden, C. 2008, ApJ, 682, 1217

Kirby, E. N., Guhathakurta, P., Simon, J. D., et al. 2010, ApJS, 191, 352

Koch, A., Feltzing, S., Adén, D., \& Matteucci, F. 2013, A\&A, 554, A5

Koch, A., \& Rich, R. M. 2014, ApJ, 794, 89

Kramida, A., Ralchenko, Y., Reader, J., \& NIST ASD Team 2014, NIST Atomic Spectra Database (ver. 5.2) (Gaithersburg, MD: National Institute of Standards and Technology) [Online], http://physics.nist.gov/asd [2014, October 17]

Kupka, F., Piskunov, N., Ryabchikova, T. A., Stempels, H. C., \& Weiss, W. W. 1999, A\&AS, 138, 119

Kurucz, R. 1993, ATLAS9 Stellar Atmosphere Programs and 2 km/s grid. Kurucz CD-ROM No. 13 (Smithsonian Astrophysical Observatory: Cambridge, MA), 13

Kurucz, R. L. 1992, RMxAA, 23, 45

Lai, D. K., Lee, Y. S., Bolte, M., et al. 2011, ApJ, 738, 51

Lee, D. M., Johnston, K. V., Sen, B., \& Jessop, W. 2015, ApJ, in press (arXiv:1410.6166)

Lee, Y. S., Suda, T., Beers, T. C., \& Stancliffe, R. J. 2014, ApJ, 788, 131

Lee, J.-W., Carney, B. W., \& Habgood, M. J. 2005, AJ, 129, 251

Lee, Y. S., Beers, T. C., Masseron, T., et al. 2013, AJ, 146, 132

Liang, Y. C., Zhao, G., \& Zhang, B. 2000, A\&A, 363, 555

Lind, K., Charbonnel, C., Decressin, T., et al. 2011, A\&A, 527, A148

Lucatello, S., Beers, T. C., Christlieb, N., et al. 2006, ApJL, 652, L37

Lucatello, S., Tsangarides, S., Beers, T. C., et al. 2005, ApJ, 625, 825

Markwardt, C. 2012, MPFIT: robust non-linear least squares curve fitting, Astrophysics Source Code Library

Marsteller, B., Beers, T. C., Rossi, S., et al. 2005, NuPhA, 758, 312

Martell, S. L., Smith, G. H., \& Briley, M. M. 2008a, PASP, 120, 839

Martell, S. L., Smith, G. H., \& Briley, M. M. 2008b, PASP, 120, 7

Martell, S. L., Smith, G. H., \& Briley, M. M. 2008c, AJ, 136, 2522

Masseron, T., Plez, B., van Eck, S., et al. 2014, A\&A, 571, A47

Mateo, M., Harris, H. C., Nemec, J., \& Olszewski, E. W. 1990, AJ, 100, 469

Matsunaga, N. 2006, PhD thesis, Univ. Tokyo

McClure, R. D., Hesser, J. E., Stetson, P. B., Vandenberg, D. A., \& Bell, R. A. 1987, AJ, 93, 1144

McClure, R. D., \& Woodsworth, A. W. 1990, ApJ, 352, 709

Mighell, K. J., \& Burke, C. J. 1999, AJ, 118, 366

Mouhcine, M. 2002, A\&A, 394, 125

Mouhcine, M., \& Lançon, A. 2003, MNRAS, 338, 572

Mould, J., \& Aaronson, M. 1979, ApJ, 232, 421

Mucciarelli, A., Bellazzini, M., Ibata, R., et al. 2012, MNRAS, 426, 2889

Newman, J. A., Cooper, M. C., Davis, M., et al. 2013, ApJS, 208, 5

Nomoto, K., Tominaga, N., Umeda, H., Kobayashi, C., \& Maeda, K. 2006, NuPhA, 777, 424

Norris, J. E., Wyse, R. F. G., Gilmore, G., et al. 2010, ApJ, 723, 1632

Orban, C., Gnedin, O. Y., Weisz, D. R., et al. 2008, ApJ, 686, 1030

Peterson, R. C., Dalle Ore, C. M., \& Kurucz, R. L. 1993, ApJ, 404, 333

Pietrzyński, G., Gieren, W., Szewczyk, O., et al. 2008, AJ, 135, 1993

Piskunov, N. E., Kupka, F., Ryabchikova, T. A., Weiss, W. W., \& Jeffery, C. S. 1995, A\&AS, 112, 525

Placco, V. M., Frebel, A., Beers, T. C., \& Stancliffe, R. J. 2014, ApJ, 797, 21

Revaz, Y., \& Jablonka, P. 2012, A\&A, 538, A82

Revaz, Y., Jablonka, P., Sawala, T., et al. 2009, A\&A, 501, 189

Rizzi, L., Held, E. V., Saviane, I., Tully, R. B., \& Gullieuszik, M. 2007, MNRAS, 380, 1255

Robertson, B., Bullock, J. S., Font, A. S., Johnston, K. V., \& Hernquist, L. 2005, ApJ, 632, 872

Salpeter, E. E. 1955, ApJ, 121, 161

Schörck, T., Christlieb, N., Cohen, J. G., et al. 2009, A\&A, 507, 817

Searle, L., \& Zinn, R. 1978, ApJ, 225, 357

Ségall, M., Ibata, R. A., Irwin, M. J., Martin, N. F., \& Chapman, S. 2007, MNRAS, 375, 831

Sharina, M., Aringer, B., Davoust, E., Kniazev, A. Y., \& Donzelli, C. J. 2012, MNRAS, 426, L31

Shetrone, M., Venn, K. A., Tolstoy, E., et al. 2003, AJ, 125, 684

Shetrone, M. D., Bolte, M., \& Stetson, P. B. 1998a, AJ, 115, 1888

Shetrone, M. D., Briley, M., \& Brewer, J. P. 1998b, A\&A, 335, 919

Shetrone, M. D., Côté, P., \& Sargent, W. L. W. 2001, ApJ, 548, 592

Shetrone, M. D., Smith, G. H., Stanford, L. M., Siegel, M. H., \& Bond, H. E. 2013, AJ, 145, 123

Sills, A., Glebbeek, E., Chatterjee, S., \& Rasio, F. A. 2013, ApJ, 777, 105

Simon, J. D., \& Geha, M. 2007, ApJ, 670, 313 
Skúladóttir, Á, Tolstoy, E., Salvadori, S., et al. 2015, A\&A, 574, AA129 Smith, G. H. 1984, AJ, 89, 801

Smith, G. H., \& Briley, M. M. 2005, PASP, 117, 895

Smith, G. H., \& Briley, M. M. 2006, PASP, 118, 740

Smith, G. H., Shetrone, M. D., Bell, R. A., Churchill, C. W., \& Briley, M. M. 1996, AJ, 112, 1511

Sneden, C., Kraft, R. P., Prosser, C. F., \& Langer, G. E. 1992, AJ, 104, 2121

Sneden, C. A. 1973, PhD thesis, Univ. Texas Austin

Sobeck, J. S., Kraft, R. P., Sneden, C., et al. 2011, AJ, 141, 175

Spite, M., Cayrel, R., Plez, B., et al. 2005, A\&A, 430, 655

Spite, M., Cayrel, R., Hill, V., et al. 2006, A\&A, 455, 291

Starkenburg, E., Shetrone, M. D., McConnachie, A. W., \& Venn, K. A. 2014, MNRAS, 441, 1217

Starkenburg, E., Hill, V., Tolstoy, E., et al. 2010, A\&A, 513, A34

Starkenburg, E., Hill, V., Tolstoy, E., et al. 2013, A\&A, 549, A88

Stetson, P. B. 2000, PASP, 112, 925

Stetson, P. B., Hesser, J. E., \& Smecker-Hane, T. A. 1998, PASP, 110, 533
Suntzeff, N. B. 1981, ApJS, 47, 1

Suntzeff, N. B., Kraft, R. P., \& Kinman, T. D. 1988, AJ, 95, 91

Takeda, Y., Kaneko, H., Matsumoto, N., et al. 2009, PASJ, 61, 563

Tinsley, B. M. 1980, FCPh, 5, 287

van Loon, J. T., van Leeuwen, F., Smalley, B., et al. 2007, MNRAS, 382,1353

VandenBerg, D. A., Bergbusch, P. A., Dotter, A., et al. 2012, ApJ, 755, 15

Venn, K. A., Irwin, M., Shetrone, M. D., et al. 2004, AJ, 128, 1177

Venn, K. A., Shetrone, M. D., Irwin, M. J., et al. 2012, ApJ, 751, 102

Villanova, S., Piotto, G., King, I. R., et al. 2007, ApJ, 663, 296

Walker, A. R. 1994, AJ, 108, 555

Wallerstein, G., \& Knapp, G. R. 1998, ARA\&A, 36, 369

Westfall, K. B., Majewski, S. R., Ostheimer, J. C., et al. 2006, AJ, 131, 375

Winnick, R. A. 2003, PhD thesis, Yale Univ.

Yanny, B., Rockosi, C., Newberg, H. J., et al. 2009, AJ, 137, 4377

Yong, D., Norris, J. E., Bessell, M. S., et al. 2013, ApJ, 762, 27

Zinn, R. 1981, ApJ, 251, 52 